\title{
On Direct Adaptive Control for Uncertain Dynamical Systems - Synthesis and Applications
}

\author{
Simon Hsu-Sheng Fu and Chi-Cheng Cheng
}

\section{Introduction}

In the rapidly growing research on nonlinear control theory, much work has been focused on the problems of uncertainties exist in the system model or systems with unknown disturbances and nonlinearities. A direct adaptive control framework for adaptive stabilization, disturbance rejection, and command following of multivariable nonlinear uncertain systems with exogenous disturbances, where the bounded disturbances were assumed to be a known vector, has developed in (Haddad \& Hayakawa, 2002) and guarantees partial stability of the closed-loop system. However, it is worth to note that the disturbances may be the result of unmodeled dynamics, noisy measurements, parameter uncertainty, or non dissipative forces affecting the plant, and most of time not available for the control design.

There are considerable amount of literatures published the area of adaptive control synthesis for uncertain systems. However, the application of Lyapunov stability theory along this track still shown relative limited results, especially

$\varepsilon$ for discrete-time systems. The major difficulty encountered concerns the proof $\stackrel{\circlearrowleft}{\circlearrowleft}$ of the global stability of the overall adaptive control loop. The main reason is that the Lyapunov candidate cannot easily be constructed, such that the negative definiteness of the Lyapunov difference could not easily shown (Zhao \& Kanellakopoulos, 1997).

For direct adaptive control gains are adjusted without explicit parameter identification. In this Chapter, we are investigating the problem of direct adaptive o control of uncertain systems, where both discrete-time and continuous-time \% systems are considered. For continuous time case, motivated by the result of \& robust stabilization of nonlinear systems affected by time-varying uniformly ¿ bounded affine disturbances (Loria et al., 1998), where a passive-based control $\bar{\Phi}$ framework has formulated and achieved global uniform convergence. Facili○े tating the direct adaptive scheme, our framework guarantees that the closed- 
loop system is Lyapunov stable under the assumption of matched disturbances. In addition, the asymptotic stable of solution $x$ with respect to origin can be proved.

There were considerable amount of discrete-time adaptive results have been published. For example, discrete-time neural net adaptive controller was depicted in (Levin \& Narendra, 1996), the MIT rule for adaptive control refers to the combination of model reference control together with a gradient type parameter update law (Mareels \& Polderman, 1996), and a stable and convergent direct adaptive control has been developed in (Johansson, 1989). An ARMARKOV model for MIMO uncertain systems achieved adaptive disturbance rejection and traction (Venugopal \& Bernstein, 1999). In addition, Shibata et al. proposed a simplified adaptive control scheme based on Lyapunov analysis while the system satisfies the so called almost strictly positive real (ASPR) condition (Shibata et al., 1996). Bar-Kana (Bar-Kana, 1989) also used ASPR assumption and presented a robust discrete-time adaptive algorithm subjected to the condition of BIBO and the boundedness of the residual term. Guo (Guo, 1997) examined the global stability for a class of discrete-time adaptive nonlinear control systems and proved critical stability for least squarebased adaptive control systems.

Furthermore, several most recent works were published and the results were close to our results presented in this Chapter. A direct adaptive control for reachable linear discrete-time systems with exogenous disturbances ( $\mathrm{Fu} \&$ Cheng, 2003, a) and $\ell_{2}$ disturbances (Fu \& Cheng, 2003, b), direct adaptive control application to a class of linear discrete-time systems, where the nominal system $A$ is known and the deviation of $\left|A-A_{c}\right|=\left|B K_{g}\right|$ is bounded, were investigated by (Fu \& Cheng, 2004, a); (Fu \& Cheng, 2004, b), and direct adaptive control for a class of nonlinear normal discrete-time systems were presented in (Fu \& Cheng, 2004, c), all results above satisfied Lyapunov stability theory. In addition, robust direct adaptive control of nonlinear uncertain systems with unknown disturbances were proposed in (Fu \& Cheng, 2005, a); (Fu \& Cheng, 2005, b). However, these solutions were limited by the hypothesis of trajectory dependence. In this paper we successfully release this limitation and obtain stability results, such that the discrete-time system stability theory (Hitz \& Anderson, 1969) can be applied.

The contents of this paper are as follows. In Section 2, we present the adaptive control framework for uncertain continuous-time nonlinear systems with matched disturbances and discrete-time systems with exogenous and $\ell_{2}$ dis- 
turbances. Next, several numerical examples are presented in Section 3, which include van der Pol oscillator, one linked rigid robot, and active suspension systems, to demonstrate the efficacy of the proposed frameworks. Finally, we illustrate the results of this paper and future research in Section 4.

\section{Adaptive Control for Uncertain Continuous-Time Nonlinear Systems with Matched Disturbances}

Our main concern in this paper is to deal with uncertain nonlinear systems perturbed by affine disturbances. We begin by considering the problem of characterizing adaptive feedback control laws for nonlinear uncertain MIMO systems $G$ given by

$$
\dot{x}=f(x(t))+G(x(t)) u(x(t))+J(x(t)) w(t, x(t))
$$

where $x(t) \in R^{n}$ is the state vector, $x(0)=x_{0}, u(t): R^{n} \rightarrow R^{m}$ is the control vector, $f: R^{n} \rightarrow R^{n}$ characterize system dynamics with uncertain entries, and $f(0)=0 . G: R^{n} \rightarrow R^{n \times m}$ and $J: R^{n} \rightarrow R^{n \times d}$ are the input and disturbance weighting matrix functions, respectively, with unknown entries. In addition, the disturbance vector $w: R \times R^{n} \rightarrow R^{d \times d}$ satisfies Assumption 2.1 illustrated next.

Assumption 2.1 (Loria et al., 1998)

The vector function $w(t, x(t))$ is bounded, and can be characterized by

$$
w(t, x(t)) \leq \bar{w}(t, x(t)) \theta_{1}+\theta_{2}
$$

where $\theta_{1} \in R^{d}$ and $\theta_{2} \in R^{d}$ are unknown constants, and $\bar{w}: R \times R^{n} \rightarrow R^{d \times d}$ is a known continuous matrix function.

It is important to note that the disturbance $w(t, x(t))$ may be the result of unmodeled dynamics, noisy measurement, parameter uncertainty or exogenous disturbances. For the nonlinear system $G$, we assume that the existence and uniqueness of solutions are satisfied and zero-state observability of (1) while 
$w(t, x(t)) \equiv 0$. Furthermore, assume there exits $F: R^{n} \rightarrow R^{s}$ with $F(0)=0$, $K_{g}: R^{n \times s}$, and $\bar{G}: R^{n} \rightarrow R^{m \times m}$ such that

$$
f_{c}(x(t)) \triangleq f(x(t))+G(x(t)) \bar{G}(x(t)) K_{g} F(x(t))
$$

is globally asymptotically stable, where a scalar function $V_{s}: R^{n} \rightarrow R$ is Lyapunov function, and $\ell: R^{n} \rightarrow R^{t}$. Then

$$
V_{s}^{\prime}(x) f_{c}(x)=-\ell^{T}(x(t)) \ell(x(t)), \forall x: R^{n} .
$$

Theorem 2.1 (Fu \& Cheng, 2005)

Consider the nonlinear uncertain system $G$ given by $(1)$ is zero state observable with $w(t, x(t)) \equiv 0$, where the disturbances $w(t, x(t))$ satisfy Assumption 2.1. In addition, let that the zero solution of (1) defined in (3) is globally asymptotically stable. Furthermore, there exists matrix functions $\Psi: R^{m \times d}$ and $\bar{J}: R^{n} \rightarrow R^{m \times m}$, such that the matching condition $G(x) \bar{J}(x) \Psi=J(x)$ is satisfied. Then the adaptive feedback control law

$$
u(x)=\bar{G}(x) K(t) F(x)+\bar{J}(x) \Phi(t)\left(\bar{w}(x, t) \hat{\theta}_{1}+\hat{\theta}_{2}\right),
$$

where $K(t): R^{m \times n}, \Phi(t): R^{m \times d}, \tilde{\theta}_{1} \underline{\underline{\Delta}} \hat{\theta}_{1}-\theta_{1}$, and $\tilde{\theta}_{2} \underline{\underline{\Delta}} \hat{\theta}_{2}-\theta_{2}$. Now, let the design matrices $P_{1}>0, P_{2}>0, Q_{1}>0, Y>0, Q_{2}>0$, and $Z>0$ with the update laws

$$
\begin{gathered}
\dot{K}=-\frac{1}{2} Q_{1} \bar{G}^{T}(x) G^{T}(x) V_{s}^{\prime T}(x) F^{T}(x) Y, \\
\dot{\Phi}=-\frac{1}{2} Q_{2} \bar{J}^{T}(x) G^{T}(x) V_{s}^{\pi T}(x)\left(\bar{w}(x, t) \hat{\theta}_{1}+\hat{\theta}_{2}\right)^{T} Z,
\end{gathered}
$$

And

$$
\begin{gathered}
\dot{\hat{\theta}}_{1}=\frac{1}{2} P_{1}^{-1} \bar{w}^{T}(x, t) J^{T}(x) V_{s}^{\prime T}(x), \\
\dot{\hat{\theta}}_{2}=\frac{1}{2} P_{2}^{-1} J^{T}(x) V_{s}^{\prime T}(x),
\end{gathered}
$$


where $V_{s}^{\prime}(x) \underline{\underline{\Delta}} \frac{\partial V_{s}(x)}{\partial x}$, guarantees that the closed-loop system, given by (1) and (5) to (9), is Lyapunov stable. In addition, if (4) is applied and let the output $y(t) \underline{\underline{\Delta}} \ell(x)$, then $\ell(x) \rightarrow 0$ as $t \rightarrow \infty$. Furthermore, the asymptotic stable solution $x$ with respect to origin will arrive when $\ell^{T}(x) \ell(x)>0$.

\section{Proof}

To show Lyapunov stability of the closed-loop system (1) and (5) to (9), we first consider the Lyapunov function candidate

$$
\begin{aligned}
& V\left(x, K, \Phi, \theta_{1}, \theta_{2}\right)= \\
& V_{s}(x)+\operatorname{tr} Q_{1}^{-1}\left(K-K_{g}\right) Y^{-1}\left(K-K_{g}\right)^{T} \\
& +\operatorname{tr} Q_{2}^{-1}(\Phi-\Psi) Z^{-1}(\Phi-\Psi)^{T}+\tilde{\theta}_{1}^{T} P_{1} \tilde{\theta}_{1}+\tilde{\theta}_{2}^{T} P_{2} \tilde{\theta}_{2}
\end{aligned}
$$

where $V_{s}(x)$ satisfies the condition of (4) and $t r$ represents trace operator. Note that the Lyapunov candidate $V\left(0, K_{g},-\Psi, 0,0\right)=0$ and $V\left(x, K, \Phi, \theta_{1}, \theta_{2}\right)>0$ for all $\left(x, K, K_{g}, \theta_{1}, \theta_{2}\right) \neq\left(0, K_{g},-\Psi, 0,0\right)$. In addition, $V\left(x, K, \Phi, \theta_{1}, \theta_{2}\right)$ is radially unbounded. Furthermore, $V\left(\bullet, K, \Phi, \theta_{1}, \theta_{2}\right)$ and $K$ are continuous in $x$ for $t \geq 0$. The corresponding Lyapunov derivative is given by

$$
\begin{aligned}
& \dot{V}=V_{s}^{\prime}(x)\left[f(x)+G(x) u(t)+J(x)\left(\bar{w}(x, t) \theta_{1}+\theta_{2}\right)\right] \\
& +2 \dot{\tilde{\theta}}_{1}^{T} P_{1} \tilde{\theta}_{1}+2 \dot{\tilde{\theta}}_{2}^{T} P_{2} \tilde{\theta}_{2}+2 \operatorname{tr} Q_{1}^{-1}\left(K-K_{g}\right) Y^{-1} \dot{K}^{T} \\
& +2 \operatorname{tr} Q_{2}^{-1}(\Phi+\Psi) Z^{-1} \dot{\Phi}^{T} \\
& =V_{s}^{\prime}(x) f(x)+V_{s}^{\prime}(x) G(x)[u(t)-\bar{G}(x) K F(x) \\
& \left.-\bar{J}(x) \Phi\left(\bar{w}(x, t) \hat{\theta}_{1}+\hat{\theta}_{2}\right)\right]+2 \operatorname{tr} Q_{2}^{-1}(\Phi+\Psi) Z^{-1} \dot{\Phi}^{T} \\
& +V_{s}^{\prime}(x) G(x) \bar{G}(x)\left(K-K_{g}\right) F(x)+2 \dot{\hat{\theta}}_{1}^{T} P_{1} \tilde{\theta}_{1} \\
& +2 \operatorname{tr} Q_{1}^{-1}\left(K-K_{g}\right) Y^{-1} \dot{K}^{T}+V_{s}^{\prime}(x) G(x) \bar{J}(x) \Phi\left(\bar{w}(x, t) \hat{\theta}_{1}+\hat{\theta}_{2}\right) \\
& -V_{s}^{\prime}(x) J(x) \bar{w}(x, t) \tilde{\theta}_{1}-V_{s}^{\prime}(x) J(x) \bar{w}(x, t) \tilde{\theta}_{2}+2 \dot{\hat{\theta}}_{2}^{T} P_{2} \tilde{\theta}_{2} \\
& =V_{s}^{\prime}(x) f_{c}(x)
\end{aligned}
$$

Next, since the condition (4) is satisfied, the resulting Lyapunov derivative along the system trajectory is

$$
\dot{V}\left(x, K, \Phi, \theta_{1}, \theta_{2}\right)=-\ell^{T}(x) \ell(x) \leq 0 .
$$


This completes the proof. Furthermore, if $\ell(x) \rightarrow 0$ as $t \rightarrow \infty$, and the asymptotic stable solution $x$ with respect to origin will arrive when $\ell^{T}(x) \ell(x)>0$.

We further extend the above result to the case where the entries of the system matrix and the input matrix are uncertain. Note that the adaptive control law (5) does not require explicit knowledge on the desire gain matrix $K_{g}$, disturbances $w(t, x(t))$, system dynamics $f(x)$, and matching matrix $\Phi$.

Theorem 2.1 also requires that the zero solution to (3) is globally asymptotically stable. Next, we consider the case where $f(x)$, input weighting matrix $G(x)=B$, and disturbance weighting matrix $J(x)=D$ are uncertain. Specifically, given as the following

$$
\dot{x}=f(x)+B u(t)+D w(x, t),
$$

where $w(t, x(t))$ satisfies Assumption 2.1, and

$$
f_{c}(x) \triangleq f(x)+B K_{g} F(x)
$$

is global asymptotically stable. Next, let $B_{s}: R^{m \times m}$ is the sign definite matrix with unknown entries; such that (Fu \& Cheng, 2004)

$$
B=\left[0_{m \times(n-m)}, B_{s}\right]^{T} \quad B_{0} \triangleq\left\{\begin{array}{c}
{\left[0_{m \times(n-m)}, I_{m}\right]^{T}, B_{s}>0} \\
{\left[0_{m \times(n-m)},-I_{m}\right]^{T}, B_{s}<0}
\end{array}\right.
$$

and

$$
B_{s} \triangleq U D_{B} U,\left|B_{s}\right|=\sqrt{B_{s}^{2}},
$$

Where $U$ is orthogonal and $D_{B}$ is real diagonal. Similarly, assume that $D_{s}: R^{d \times d}$ is the sign definite matrix with unknown matrix; that is

$$
D=\left[0_{d \times(n-d)}, D_{s}\right]^{T} D_{0} \triangleq\left\{\begin{array}{c}
{\left[0_{d \times(n-d)}, I_{d}\right]^{T}, D_{s}>0} \\
{\left[0_{d \times(n-d)},-I_{d}\right]^{T}, D_{s}<0}
\end{array}\right.
$$

and 


$$
D_{s} \underline{\underline{\Delta}} \hat{U} D_{D} \hat{U},\left|D_{s}\right|=\sqrt{D_{s}^{2}}
$$

\section{Corollary 2.1}

Consider the nonlinear uncertain system given by (13) is zero state observable. Let $B$ and $D$ satisfy (15) and (17), respectively. Then, the adaptive feedback control law

$$
u(x)=K(t) F(x)+\Phi(t)\left(\bar{w}(x, t) \hat{\theta}_{1}+\hat{\theta}_{2}\right)
$$

with the update laws

$$
\begin{gathered}
\dot{K}=-\frac{1}{2} B_{0}^{T} V_{s}^{\prime T}(x) F^{T}(x) Y, \\
\dot{\Phi}=-\frac{1}{2} B_{0}^{T}(x) V_{s}^{\prime T}(x)\left(\bar{w}(x, t) \hat{\theta}_{1}+\hat{\theta}_{2}\right)^{T} Z,
\end{gathered}
$$

and

$$
\begin{gathered}
\dot{\hat{\theta}}_{1}=\frac{1}{2} \bar{w}^{T}(x, t) D_{0}^{T} V_{s}^{\prime T}(x), \\
\dot{\hat{\theta}}_{2}=\frac{1}{2} D_{0}^{T} V_{s}^{\prime T}(x),
\end{gathered}
$$

guarantees that the closed-loop system, given by (13), (19), and (20) to (23) is Lyapunov stable. Furthermore, if (14) is applied and let the output $y(t) \underline{\underline{\Delta} \ell}(x)$, then $\ell(x) \rightarrow 0$ as $t \rightarrow \infty$. Furthermore, the asymptotic stable solution $x$ with respect to origin will arrive when $\ell^{T}(x) \ell(x)>0$.

\section{Proof}

The result is a direct extension of Theorem 2.1. Let $G(x)=I_{m}$ and $\hat{J}(x)=I_{m}$, and the matching condition be $B \hat{J}(x) \Psi=D$. In addition, let $P_{1}>0, P_{2}>0$, $Q_{1}>0, Q_{2}>0, Y>0, Z>0$, and assume that $P_{1}^{-1} \bar{w}^{T}(x, t)=\bar{w}^{T}(x, t) P_{1}^{-1}$, and let 
$Q_{1}$ be replaced by $q_{1}\left|B_{s}\right|^{-1}, Q_{2}$ be replaced by $q_{2}\left|B_{s}\right|^{-1}, P_{1}$ be replaced by $q_{3}\left|D_{s}\right|$, and $P_{2}$ be replaced by $q_{4}\left|D_{s}\right|$, where $q_{i}>0, i=1,2,3,4$ are arbitrary real. Next, let $q_{1} Y$ and $q_{2} Z$ be replaced by $Y$ and $Z$ respectively. Finally, let $q_{3}=q_{4}=1$, then the resulting update laws (20) and (21) are obtained.

Note that the frameworks of Theorem 2.1 and Corollary 2.1 can extend to linear systems such that $f(x)=A x$ and $f_{c}(x)=A_{c} x$, where $A_{c}=A+B K_{g}$ is an asymptotically stable matrix. Also, applied to nonlinear time-varying uncertain systems given by

$$
\dot{x}=f(x(t), t)+G(x(t), t) u(x(t))+J(x(t), t) w(t, x(t))
$$

and tracking problems given by

$$
\dot{e}=f(e(t))+G(e(t)) u(e(t))+J(e(t)) w(t, e(t))
$$

where $e(t)=x(t)-r_{d}(t)$ is tracking error, and $r_{d}(t)$ is reference. Next, we present the discrete-time counterpart of direct adaptive control for Uncertain Nonlinear Systems given as Section 3.

\section{Adaptive Control Designs for Nonlinear Uncertain Discrete-Time Systems}

\subsection{Discrete-Time Systems with Disturbance Measurement}

In this section, we extend the results of Theorem 2.1 to nonlinear uncertain discrete time MIMO systems with disturbances measurement given by

$x(k+1)=f(x(k))+G(x(k)) u(x(k))+J(x(k)) w(k)$,

which is zero state observable when $w(k) \equiv 0$, where $x(k) \in R^{n}$ is the state vector, $u(k): R^{n} \rightarrow R^{m}$ is the control vector, $f: R^{n} \rightarrow R^{n}$ characterize system dynamics with uncertain entries, and $f(0)=0 . G: R^{n} \rightarrow R^{n \times m}$ and $J: R^{n} \rightarrow R^{n \times d}$ are the input and disturbance weighting matrix functions, respectively. In ad- 
dition, let the disturbance vector $w: R \times R^{n} \rightarrow R^{d \times d}$ is measurable, then the feedback law given by

$$
u(x(k))=\bar{G}(x(k)) K(k) F(x(k))+\bar{J}(x(k)) \Phi(k) w(k)
$$

where $K(k): R^{m \times n}, \Phi(k): R^{m \times d}$, and $F: R^{n} \rightarrow R^{s}$.

Theorem 3.1 (Fu \& Cheng, 2005)

Consider the nonlinear discrete time MIMO systems with exogenous disturbances given by (26). Next, assume there exist $\hat{J}: R^{n} \rightarrow R^{n \times d}$ and $\Psi: R^{m \times d}$, such that the matching condition $G(x) \hat{J}(x) \Psi=J(x)$ is satisfied. Furthermore, let $\quad P_{1 u}: R^{n} \rightarrow R^{1 \times m}, \quad P_{2 u}: R^{n} \rightarrow R^{m \times m}, \quad P_{u w}: R^{n} \rightarrow R^{m \times d}, \quad P_{1 w}: R^{n} \rightarrow R^{1 \times d}$, and $P_{2 w}: R^{n} \rightarrow R^{d \times d}$, the Lyapunov function $V_{s}$ is defined as

$$
\begin{aligned}
& V_{s}(x(k+1)) \underline{\Delta} V_{s}(f(x))+P_{1 u}(x) u(x)+u^{T}(x) P_{2 u}(x) u(x) \\
& +u^{T}(x) P_{u w}(x) w(k)+P_{1 w}(x) w(k)+w^{T}(k) P_{2 w}(x) w(k),
\end{aligned}
$$

In addition, let $\Gamma: R^{n} \times R^{d} \rightarrow R$ is a positive scalar function, $\ell: R^{n} \rightarrow R^{p}$ is output vector, then

$$
0=V_{s}\left(f_{c}(x)\right)-V_{s}(x)+\ell^{T}(x) \ell(k)+\Gamma(x, w) .
$$

The adaptive feedback control law (27), with the measurable disturbances, and the update laws

$$
\begin{aligned}
& K(k+1)=K(k)-Q_{1} \hat{G}^{T}(x(k)) G^{T}(x(k)) P G(x(k)) \\
& {\left[2 K(k) F(x(k)) F^{T}(x(k))+\hat{J}(x(k)) \Phi(k) w(k) F^{T}(x(k))\right] Y,} \\
& \Phi(k+1)=\Phi(k)-Q_{2} \hat{G}^{T}(x(k)) G^{T}(x(k)) P G(x(k)) G^{T}(x(k)) P \\
& {[J(x(k))-2 G(x(k)) \hat{J}(x(k)) \Phi(x(k))] w(k) w^{T}(k) Z,}
\end{aligned}
$$

where $Q_{1}>0, Y>0, Q_{2}>0$, and $Z>0$, guarantees that the closed-loop system given by (26), (27), (30) and (31) is Lyapunov stable. 


\section{Proof}

To show Lyapunov stability of the closed-loop system, given by (26), (27), (30) and (31). We first consider the Lyapunov function candidate

$$
\begin{aligned}
& V(x(k), K(k), \Phi(k))= \\
& V_{s}(x(k))+\operatorname{tr} Q_{1}\left(K(k)-K_{g}\right) Y^{-1}\left(K(k)-K_{g}\right)^{T} \\
& +\operatorname{tr} Q_{2}(\Phi(k)-\Psi) Z^{-1}(\Phi(k)-\Psi)^{T},
\end{aligned}
$$

Note that the Lyapunov candidate $V\left(0, K_{g}, \Psi\right)=0$, and $V(x, K, \Phi)>0$ for all $(x, K, \Phi) \neq\left(0, K_{g}, \Psi\right)$. In addition, $V(\bullet, K, \Phi)$ and $K$ are continuous with respect to $x, V(x, K, \bullet)$ and $\Phi$ are continuous with respect to $w$ for $k \geq 1$. Let $x(k)$, $k \geq 0$, denotes the solution of the closed-loop system (26) and (27), and is global asymptotic stability when $w(k) \equiv 0$. The corresponding Lyapunov difference is given by

$$
\begin{aligned}
& \Delta V(k)=\Delta V(x(k), K(k), \Phi(k))= \\
& V(x(k+1), K(k+1), \Phi(k+1)-V(x(k), K(k), \Phi(k)),
\end{aligned}
$$

and follow the similar proof of Theorem 2.1 with the following adaptive laws

$$
\begin{gathered}
K(k+1)=K(k)-Q_{1} \hat{F}(x(k), w(k)) F^{T}(x(k)) Y, \\
\Phi(k+1)=\Phi(k)-Q_{2} R_{w}(w(k)) w^{T}(k) Z,
\end{gathered}
$$

where

$$
\begin{gathered}
\hat{F}(x, w)=\frac{1}{2} \hat{G}^{T}(x)\left[P_{1 u}^{T}(x)+P_{2 u}(x) \hat{G}(x) K(k) F(x)\right. \\
\left.+P_{2 u}(x) \hat{J}(x) \Phi(k) w(k)\right], \\
R_{w}(w(k))=-\frac{1}{2} \hat{J}^{T}(x(k))\left[P_{u w}(x(k))\right. \\
\left.+G^{T}(x(k)) P J(x(k))\right] w(k),
\end{gathered}
$$


Next, let $P=N^{T} N, N: R^{n \times n}$, and chose the following

$$
\begin{gathered}
P_{u w}(x(k))=-4 P_{2 u}(x(k)) \hat{J}(x(k)) \Phi(k), \\
P_{1 u}(x(k))=2 F^{T}(x(k)) K^{T}(k) \hat{G}^{T}(x(k)) P_{2 u}(x(k)), \\
P_{2 u}(x(k))=G^{T}(x(k)) P G(x(k)), \\
P_{1 w}(x(k))= \\
2 w^{T}(k) \Phi^{T}(k) \hat{J}^{T}(x(k)) P_{2 u}(x(k)) \hat{J}(x(k)) \Phi(k), \\
P_{2 w}(x(k))=\left[G^{T}(k) \hat{J}^{T}(x(k)) \Phi(k)-J(x(k))\right]^{T} \\
P\left[G^{T}(k) \hat{J}^{T}(x(k)) \Phi(k)-J(x(k))\right],
\end{gathered}
$$

by substituting (34) and (35) into (33), after some manipulations yields

$$
\begin{aligned}
& \Delta V(k)=\left[w^{T}(k) Z w(k)\right] R_{w}^{T}(x) Q_{2} R_{w}(x)+ \\
& {\left[F^{T}(x) Y F(x)\right] \hat{F}^{T}(x) Q_{1} \hat{F}(x)+V_{s}\left(f_{c}(x)\right)-V_{s}(x)} \\
& -w^{T}(k) \Phi^{T}(k) \hat{J}^{T}(x) P_{2 u}(x) \hat{J}(x) \Phi(k) w(k) \\
& 2 w^{T}(k) \Phi^{T}(k) \hat{J}^{T}(x) P_{2 u}(x) \hat{J}(x) \Phi(k) F(x) \\
& -\Gamma_{F}^{T}(x) G^{T}(x) P G(x) \Gamma_{F}(x), \\
& \Gamma_{F}(x(k))=\hat{G}^{T}(x(k))\left[K(k)-K_{g}\right] F(x(k)),
\end{aligned}
$$

Since (29) is satisfied, and let

$$
\begin{aligned}
& \Delta V(k)=-\left|N G(x) \hat{G}(x)\left[K(k)-K_{g}\right] F(x)+N G(x) \hat{J}(x) \Phi(k) w(k)\right|_{2}^{2} \\
& -\ell^{T}(x) \ell(x) \leq-\ell^{T}(x) \ell(x) .
\end{aligned}
$$

where $x(k)$ denotes the solution to the closed-loop dynamical system (26) and (27). Then the resulting Lyapunov difference becomes 
This completes the proof. If $\ell(x) \neq 0, k \geq 0$, then $x \rightarrow 0$ as $k \rightarrow \infty$. Furthermore, if $\ell(x) \rightarrow 0$ as $k \rightarrow \infty$, and the asymptotic stable solution $x$ with respect to origin will arrive when $\ell^{T}(x) \ell(x)>0$.

Note that the adaptive control laws (30) and (31) do not require explicit knowledge of the matrix $K_{g}$, the disturbance matching matrix $\Psi$ and system dynamics $f(x(k))$. Next, we extend the solution of Theorem 3.1 to the following dynamic system

$$
x(k+1)=f(x(k))+B u(x(k))+D w(k),
$$

where the entries of $B$ and $D$ are unknown and satisfy the conditions given in (15) and (17), respectively.

\section{Corollary 3.1}

Consider the nonlinear discrete time system given by (44). Next, let $F: R^{n} \rightarrow R^{s}$ and there exists $K_{g}: R^{n \times s}$ such that $f_{c}(x) \underline{\underline{\Delta}} f(x)+B K_{g} F(x)$ is exponentially stable. In addition, let $\Psi: R^{m \times d}$ and the matching condition $B \Psi=D$ is satisfied. Then the feedback law

$$
u(x(k))=K(k) F(x(k))+\Phi(k) w(k),
$$

with the adaptive gain matrices

$$
\begin{aligned}
& K(k+1)=K(k)-q^{2} B_{0}^{T} P B_{0}[2 K(k) F(x(k)) \\
& +\Phi(k) w(k)] F^{T}(x(k)) Y, \\
& \Phi(k+1)=\Phi(k)-q^{2} B_{0}^{T} P\left[2 B_{0} \Phi(k)-D_{0}\right] w(k) w^{T}(k),
\end{aligned}
$$

where $K(k)=\left|B_{s}\right| K(k), \Phi(k)=\left|B_{s}\right| \Phi(k)\left|D_{s}\right|^{-1}, w(k)=\left|D_{s}\right| w(k)$, and $q>0$, guarantees that the closed-loop system given by (44), (45), (46), and (47) is Lyapunov stable, and equivalent to the following

$$
x(k+1)=f(x(k))+B_{0} K(k) F(x(k))+\left(B_{0} \Phi(k)+D_{0}\right) w(k),
$$




\section{Proof}

The proof is a direct extension of Theorem 3.1. First, we consider the Lyapunov candidate given by (32), the feedback law (45), with the assumptions that (15), (17), (28) and (29) are satisfied. Next, consider the following adaptive laws

$$
\begin{aligned}
&\left|B_{s}\right| K(k+1)=\left|B_{s}\right| K(k)+\left|B_{s}\right| Q_{1}\left|B_{s}\right| B_{0}^{T} P B_{0} \Phi(k)\left|D_{s}\right|^{-1}\left|D_{s}\right| w(k) F^{T}(x(k)) Y \\
&-2\left|B_{s}\right| Q_{1}\left|B_{s}\right| B_{0}^{T} P B_{0} K(k) F(x(k)) F^{T}(x(k)) Y, \\
&\left|B_{s}\right| \Phi(k+1)\left|D_{s}\right|^{-1}=\left|B_{s}\right| Q_{2}\left|B_{s}\right| B_{0}^{T} P D_{0}\left|D_{s}\right|\left|D_{s}\right|^{-1} \Phi_{w}(k)\left|D_{s}\right|^{-1} Z\left|D_{s}\right|^{-1} \\
&+\left|B_{s}\right| \Phi(k)\left|D_{s}\right|^{-1}-2\left|B_{s}\right| Q_{2}\left|B_{s}\right| B_{0}^{T} P B_{0} \Phi(k)\left|D_{s}\right|^{-1} \Phi_{w}(k)\left|D_{s}\right|^{-1} Z\left|D_{s}\right|
\end{aligned}
$$

Where

$$
\begin{gathered}
\Phi_{w}(k)=\left|D_{s}\right| w(k) w^{T}(k)\left|D_{s}\right|, \\
Q_{1}=Q_{2}=q^{2}\left|B_{s}\right|^{-1}\left|B_{s}\right|^{-1}, \\
Z=\left|D_{s}\right|\left|D_{s}\right|,
\end{gathered}
$$

The resulting Lyapunov difference becomes

$$
\Delta V(k) \leq-\ell^{T}(x) \ell(x)
$$

Then (49) and (50) reduce to (46) and (47), respectively. This complete the proof. Finally, since the adaptive gains we obtained are actually $\left|B_{s}\right| K(k)$ and $\left|B_{s}\right| \Phi(k)\left|D_{s}\right|^{-1}$, and the measured disturbance is $\left|D_{s}\right| w(k)$. The closed-loop system given by (44), (45), (46) and (47) can be rewritten as (48).

Lastly, we propose a robust adaptive solution to the linear uncertain systems given as following

$$
x(k+1)=A x(k)+B u(x(k))+D w(k),
$$


where $B$ and $D$ matrices satisfy the conditions given by (15) and (17), pair $(A, B)$ is controllable, and there exists a gain matrix $K_{g}: R^{m \times n}$, such that $A_{c}=A+B K_{g}$ is exponentially stable. In addition, let $\Delta A=A_{c}-A$ is bounded, and the norm $|\Delta A|$ indicates the system dynamics $A$ deviates from the stable solution $A_{c}$ (Fu \& Cheng, 2004).

\section{Corollary 3.2}

Consider the nonlinear discrete time system given by (52). Assume that $B$ and $D$ satisfy (15) and (17), respectively. Next, let $\Psi: R^{m \times d}$ and the matching condition $B \Psi=-D$ is satisfied. The feedback law given by

$$
u(x(k))=K(k) x(k)+\Phi(k) w(k)
$$

where $K(k)=\left|B_{s}\right| K(k), \Phi(k)=\left|B_{s}\right| \Phi(k)\left|D_{s}\right|^{-1}, w(k)=\left|D_{s}\right| w(k)$, and $q>0$. Furthermore, the adaptive gain matrices

$$
\begin{aligned}
& K(k+1)=K(k)-q^{2} B_{0}^{T} P\left[\left(B_{0} K(k)+A_{c}\right) x(k)\right. \\
& \left.+\left(B_{0} \Phi(k)+D_{0}\right) w(k)\right] x^{T}(k) Y, \\
& \Phi(k+1)=\Phi(k)+q^{2} B_{0}^{T} P\left[\left(B_{0} \Phi(k)\right.\right. \\
& \left.\left.-D_{0}\right) w(k)-A_{c} x(k)\right] w^{T}(k),
\end{aligned}
$$

guarantees that the closed-loop system given by (52), (53), (54), and (55) is Lyapunov stable, and equivalent to the following form

$$
x(k+1)=\left(A+B_{0} K(k)\right) x(k)+\left(B_{0} \Phi(k)+D_{0}\right) w(k) .
$$

\section{Proof}

The proof is a direct extension of Corollary 3.1. First, we consider the Lyapunov function candidate

$$
\begin{aligned}
V(x(k), K(k), \Phi(k))= & x^{T}(k) P x(k)+\operatorname{tr} Q_{1}\left(K(k)-K_{g}\right) Y^{-1}\left(K(k)-K_{g}\right)^{T} \\
& +\operatorname{tr} Q_{2}(\Phi(k)-\Psi) Z^{-1}(\Phi(k)-\Psi)^{T},
\end{aligned}
$$


Next, consider the Lyapunov difference (33), and assume that (15), (17), (28) and (29) are satisfied. Then the feedback control (53) with the adaptive laws given by

$$
\begin{aligned}
& \left|B_{s}\right| K(k+1)=\left|B_{s}\right| K(k)-\left|B_{s}\right| Q_{1}\left|B_{s}\right| B_{0}^{T} P\left[B_{0}\left|B_{s}\right| K(k)\right. \\
& \left.+A_{c}\right] x(k) x^{T}(k) Y \quad-\left|B_{s}\right| Q_{1}\left|B_{s}\right| B_{0}^{T} P\left[B_{0}\left|B_{s}\right| \Phi(k)\left|D_{s}\right|^{-1}\right. \\
& \left.+D_{0}\right]\left|D_{s}\right| w(k) x^{T}(k) Y,
\end{aligned}
$$

$\left|B_{s}\right| \Phi(k+1)\left|D_{s}\right|^{-1}=-\left|B_{s}\right| Q_{2}\left|B_{s}\right| B_{0}^{T} P A_{c} x(k) w^{T}(k)\left|D_{s}\right|\left|D_{s}\right|^{-1} Z\left|D_{s}\right|^{-1}$

$-\left|B_{s}\right| Q_{2}\left|B_{s}\right| B_{0}^{T} P\left[D_{0}+B_{0}\left|B_{s}\right| \Phi(k)\left|D_{s}\right|^{-1}\right]\left|D_{s}\right| w(k) w^{T}(k)\left|D_{s}\right|\left|D_{s}\right|^{-1} Z\left|D_{s}\right|^{-1}$

$+\left|B_{s}\right| \Phi(k)\left|D_{s}\right|^{-1}$,

After some manipulations, the Lyapunov difference $\Delta V$ reduced to

$$
\begin{aligned}
& \Delta V(k)=x^{T}(k)\left[A_{c}^{T} P A_{c}-P+K_{g}^{T} B^{T} P B K_{g}\right. \\
& +R_{w}^{T}(x(k), w(k)) Q_{2} R_{w}(x(k), w(k)) Z+ \\
& \left.\hat{F}^{T}(x(k), w(k)) Q_{1} \hat{F}(x(k), w(k))\right] x(k) \\
& -x^{T}(k) K^{T}(k) B^{T} P B K(k) x(k)-|N B \Phi(k) w(k)+N D w(k)|_{2}^{2},
\end{aligned}
$$

where

$$
\begin{gathered}
\hat{F}(x(k), w(k))=B^{T} P[(B K(k)+ \\
\left.\left.A_{c}\right) x(k)+(B \Phi(k)+D) w(k)\right], \\
R_{w}(x(k), w(k))=B^{T} P\left[A_{c} x(k)\right. \\
+(B \Phi(k)+D) w(k)],
\end{gathered}
$$

Since $x(k)$ be the solution of the closed-loop system, and the following conditions are satisfied

$$
\begin{gathered}
|\Delta A|^{2} P \geq \Delta A^{T} P \Delta A=K_{g}^{T} B^{T} P B K_{g} \\
R \geq \hat{F}(x(0), \hat{w}) Y+R_{w}^{T}(x(0), \hat{w}) Q_{2} R_{w}(x(0), \hat{w}) Z+|\Delta A|^{2} P
\end{gathered}
$$


The resulting Lyapunov difference becomes

$$
\Delta V(k) \leq-\ell^{T}(x) \ell(x) .
$$

Next, let $Q_{1}=Q_{2}=q^{2}\left|B_{s}\right|^{-1}\left|B_{s}\right|^{-1}$ and $Z=\left|D_{s}\right|\left|D_{s}\right|$, then (58) and (59) reduce to (54) and (55), respectively. In addition, since a normalized adaptive gains $\left|B_{s}\right| K(k)$ and $\left|B_{s}\right| \Phi(k)\left|D_{s}\right|^{-1}$ are obtained through this design, with measured disturbance $w(k)$. The closed-loop system given by (52), (53), (54) and (55) can be rewritten as (48). This completes the proof.

Note that, the framework of of Corollary 3.1 and Corollary 3.2 do not require the knowledge of $\left|B_{s}\right|$ and $\left|D_{s}\right|$.

\subsection{Discrete-Time Systems with $\ell_{2}$ Disturbances}

In this section we propose an adaptive feedback control solution for nonlinear uncertain discrete time MIMO systems with bounded $\ell_{2}$ disturbances given by

$$
\begin{aligned}
& x(k+1)=f(x(k)) \\
& +G(x(k)) u(x(k))+J(x(k)) w(k),
\end{aligned}
$$

where $w: R^{d}, k \geq 1$, is the unknown bounded energy $\ell_{2}$ disturbance, $x(k) \in R^{n}$ is the state vector, $u(k): R^{n} \rightarrow R^{m}$ is the control vector, $f: R^{n} \rightarrow R^{n}$ characterize system dynamics with uncertain entries, and $f(0)=0 . G: R^{n} \rightarrow R^{n \times m}$ and $J: R^{n} \rightarrow R^{n \times d}$ are the input and disturbance weighting matrix functions, respectively. and the feedback law

$$
u(x(k))=\hat{G}(x(k)) K(k) F(x(k)),
$$

guarantees nonexpansivity condition given as Theorem 4.1.

\section{Theorem 4.1}

A nonlinear discrete-time system (66) is nonexpansive when $x(0)=x_{0}$, if the solution $x(k), k \geq 0$, satisfies the following 


$$
\sum_{i=0}^{k} z^{T}(i) z(i) \leq \hat{\gamma}^{2} \sum_{i=0}^{k} w^{T}(i) w(i)+V(x(0), K(0)),
$$

where $z(k)$ is output signal, and the Lyapunov candidate

$$
\begin{aligned}
& V(x(k), K(k))=V_{s}(x(k))+ \\
& \operatorname{tr} Q_{1}\left(K(k)-K_{g}\right) Y^{-1}\left(K(k)-K_{g}\right)^{T},
\end{aligned}
$$

for all $k: \mathrm{N}, w(\bullet) \in \ell_{2}, D: R^{n \times d}, \hat{\gamma}$ and $\gamma$ be positive reals such that $\hat{\gamma}^{2} I_{d} \geq \gamma^{2} I_{d}+2 D^{T} P D$.

Next, we state and prove the discrete-time adaptive result for nonlinear system with bounded energy $\ell_{2}$ disturbances.

\section{Theorem 4.2}

Consider the nonlinear discrete time system $G$ given by (66), where the system dynamics $f$ is uncertain. Next, We assume that there exists a gain matrix $K_{g} \in R^{m \times s}, \hat{G}: R^{n} \rightarrow R^{m \times m}$, and vector $F: R^{n} \rightarrow R^{s}$, such that

$$
f_{c}(x(k))=f(x(k))+G(x(k)) \hat{G}(x(k)) K_{g} F(x(k)),
$$

Furthermore, there exist $P_{1 u}: R^{n} \rightarrow R^{1 \times m}, \quad P_{2 u}: R^{n} \rightarrow R^{m \times m}, \quad P_{u w}: R^{n} \rightarrow R^{m \times d}$, $P_{1 w}: R^{n} \rightarrow R^{1 \times d}$, and $P_{2 w}: R^{n} \rightarrow R^{d \times d}$, the Lyapunov function $V_{s}$ is defined as

$$
\begin{aligned}
& V_{s}(x(k+1)) \underline{\Delta} V_{s}(f(x(k)))+P_{1 u}(x(k)) u(x(k))+u^{T}(x(k)) P_{2 u}(x(k)) u(x(k)) \\
& +u^{T}(x(k)) P_{u w}(x(k)) w(k)+P_{1 w}(x(k)) w(k)+w^{T}(k) P_{2 w}(x(k)) w(k),
\end{aligned}
$$

Let $\Gamma: R^{n} \rightarrow R$ be a positive scalar function and $\ell: R^{n} \rightarrow R^{p}$ is output vector, the following is assumed to be true

$$
0=V_{s}\left(f_{c}(x(k))\right)-V_{s}(x(k))+\ell^{T}(x(k)) \ell(x(k))+\Gamma(x(k)),
$$

Then the adaptive feedback control law

$$
u(x(k))=\hat{G}(x(k)) K(k) F(x(k)),
$$


with the update law

$$
\begin{aligned}
& K(k+1)=K(k)-\frac{1}{2} Q \hat{G}(x(k)) P_{1 u}(x(k)) F(x(k)) Y \\
& -Q \hat{G}(x(k)) P_{2 u}(x(k)) \hat{G}(x(k)) K(k) F(x(k)) F^{T}(x(k)) Y,
\end{aligned}
$$

where $Q>0$ and $Y>0$, guarantees that the closed-loop system, given by (66), (73), and (74), satisfies the nonexpansivity constraint given in Theorem 4.1.

\section{Proof}

The proof is a direct extension of Theorem 2.1 and Theorem 4.1. We first consider the Lyapunov function candidate (69), such that $V\left(0, K_{g}\right)=0$, and $V(x(k), K(k))>0$ for all $(x(k), K(k)) \neq\left(0, K_{g}\right)$, then $V(x(k), K(k))$ is radially unbounded. Furthermore, assume that $V(\bullet, K(k))$ and $K(k)$ are continuous in $x(k)$ for $k \geq 1$. The corresponding Lyapunov difference is given by

$$
\Delta V(k)=V(x(k+1), K(k+1))-V(x(k), K(k)) .
$$

Next, consider the update law

$$
\begin{aligned}
& K(k+1)=K(k)-Q \hat{F}(x(k)) F^{T}(x(k)) Y, \\
& \hat{F}(x(k))=\frac{1}{2} \hat{G}^{T}(x(k)) P_{1 u}(x(k))+\hat{G}^{T}(x(k)) \\
& P_{2 u}(x(k)) \hat{G}(x(k)) K(k) F(x(k)),
\end{aligned}
$$

we then add and subtract $\gamma^{2} w^{T}(k) w(k)$ to and from (75), and apply the fact $\operatorname{tr} x y^{T}=y^{T} x, \forall x, y \in R^{n}$, then (75) becomes

$$
\begin{aligned}
\Delta V(k)= & V_{s}\left(f_{c}(x(k))\right)-V_{s}(x(k))-F^{T}(x) K_{g}^{T} \hat{G}^{T}(x) P_{2 u}(x) \hat{G}(x) K_{g} F(x) \\
& -F^{T}(x) K^{T}(x) \hat{G}^{T}(x) P_{2 u}(x) \hat{G}(x) K(x) F(x)+w^{T}(k) P_{2 w}(x) \\
& +2 F^{T}(x) K_{g}^{T} \hat{G}^{T}(x) P_{2 u}(x) \hat{G}(x) K(x) F(x)+P_{1 w}(x) w(k) \\
& +F^{T}(x) K^{T}(x) \hat{G}^{T}(x) P_{u w}(x) w(k)+\left[F^{T}(x) Y F(x)\right] \hat{F}^{T}(x) Q \hat{F}(x)
\end{aligned}
$$


Furthermore, let

$$
\begin{aligned}
\Gamma(x(k)) & =\left[F^{T}(x) Y F(x)\right] \hat{F}^{T}(x) Q \hat{F}(x) \\
+ & F^{T}(x) K^{T}(k) \hat{G}^{T}(x) P_{2 u}(x) \hat{G}(x) K(x) F(x), \\
P_{2 u}(x) & =G^{T}(x) P G(x), \\
P_{2 w}(x) & =J^{T}(x) P J(x), \\
P_{u w}(x) & =2 G^{T}(x) P J(x), \\
P_{1 w}(x) & =\gamma^{2} w^{T}(k), \quad \gamma^{2} I_{d}-P_{2 w}(x) \geq 0,
\end{aligned}
$$

and $P=N^{T} N$. After some manipulations, the resulting Lyapunov difference becomes

$$
\begin{gathered}
\Delta V(k) \leq-\ell^{T}(x(k)) \ell(x(k))+\gamma^{2} w^{T}(k) w(k) \\
\rightarrow V(x(k), K(k))-V(x(0), K(0)) \leq-\sum_{i=0}^{k} \ell^{T}(x(i)) \ell(x(i))+\gamma^{2} \sum_{i=0}^{k} w^{T}(i) w(i) \\
\rightarrow-\sum_{i=0}^{k} \ell^{T}(x(i)) \ell(x(i)) \leq \gamma^{2} \sum_{i=0}^{k} w^{T}(i) w(i)+V(x(0), K(0))-V(x(k), K(k)) \\
\leq \gamma^{2} \sum_{i=0}^{k} w^{T}(i) w(i)+V(x(0), K(0))
\end{gathered}
$$

This proves that the closed-loop trajectory satisfies the nonexpansivity constraint given in Theorem 4.1. In addition, if $\ell(x(k)) \neq 0, k \geq 0$, then $x(k) \rightarrow 0$ as $k \rightarrow \infty, \forall x(0) \in R^{n}$. Finally, combining (78) and (76), (74) can therefor be obtained.

Next, let $G(x(k))=B$ is sign definiteness matrix and satisfies (15). Specifically, the nonlinear system given by

$$
x(k+1)=f(x(k))+B u(x(k))+J(x(k)) w(k) .
$$

We state without proof the following Corollary, since this is a direct extension of Theorem 4.2.

\section{Corollary 4.1}

Consider the nonlinear discrete time system given by (80). Assume that $F: R^{n} \rightarrow R^{s}$ and $\Gamma: R^{n} \times R^{d} \rightarrow R$, such that (72) is applied, and $V_{s}$ is defined as (71). The feedback law 


$$
u(x(k))=K(k) F(x(k)),
$$

with the normalized adaptive gain matrices

$$
K(k+1)=K(k)-2 q^{2} B_{0}^{T} P B_{0} K(k) F(x(k)) F^{T}(x(k)) Y,
$$

where $K(k)=\left|B_{s}\right| K(k)$, and $q>0$, guarantees that the closed-loop system given by (80), (81), and (82), equivalent to

$$
x(k+1)=f(x(k))+B_{0} K(k) F(x(k))+J(x(k)) w(k),
$$

satisfies the nonexpansivity constraint given in Theorem 4.1.

Note that the solution of adaptive gain matrix (82) is given by the selection of

$$
P_{1 u}(x(k))=2 B^{T} P B K(k) F(x(k)) \text {. }
$$

Specifically, if $P_{1 u}(x(k))=2 B^{T} P B x(k)$, then the adaptive gain matrix can be given by

$$
\begin{aligned}
& K(k+1)=K(k)-q^{2} B_{0}^{T} P\left(B_{0} K(k)\right. \\
& F(x(k))+x(k)) F^{T}(x(k)) Y,
\end{aligned}
$$

Finally, we consider the linear discrete-time system $G$, where $J(x(k))=D$ is a sign definiteness matrix and $f(x(k))=A x(k)$. Specifically, given by

$$
x(k+1)=A x(k)+B u(x(k))+D w(k),
$$

where $A \in R^{n \times n}$ is the time-invariant uncertain system matrix, $B \in R^{n \times m}$ is the input matrix, and $D \in R^{n \times d}$ is the disturbance weighting matrix. Let $(A, B)$ be controllable pair, and $B$ and $D$ satisfy (15) and (17), respectively. We then state and prove the robust adaptive control design for linear uncertain systems as following.

\section{Corollary 4.2}

Consider the reachable linear discrete time system $G$ given by (86). Assume there exists a gain matrix $K_{g}: R^{m \times n}$, such that $A_{c}=A+B K_{g}$ is exponentially stable, and let $\Delta A=A_{c}-A$ is bounded, and the norm $|\Delta A|$ indicates the system dynamics $A$ deviates from the stable solution $A_{c}$. Next, let $\gamma$ be a posi- 
tive real, $L \in R^{n \times d}, W \in R^{d \times d}, R>0, \hat{R}>0, \hat{\Gamma}>0$, and $P \in R^{n \times n}$ be the positive definite solution to the discrete-time Lyapunov equation given as

$$
\begin{gathered}
A_{c}^{T} P A_{c}-P=-\hat{\Gamma}-R \hat{R}, \\
A_{c}^{T} P D=L W, \\
\gamma^{2} I_{d}-2 D^{T} P D=W^{T} W,
\end{gathered}
$$

Then the adaptive feedback control as (81), with the update law

$$
K(k+1)=K(k)-q^{2} B_{0}^{T} P\left(B_{0} K(k)+A_{c}\right) x(k) x^{T}(k) Y,
$$

guarantees that the closed-loop system, given by (86), (81), and (90), satisfies the nonexpansivity constraint given in Theorem 4.1.

\section{Proof}

We first consider the Lyapunov function candidate given by

$$
\begin{aligned}
& V(x(k), K(k))=x^{T}(k) P x(k) \\
& +\operatorname{tr} Q^{-1}\left(K(k)-K_{g}\right) Y^{-1}\left(K(k)-K_{g}\right)^{T},
\end{aligned}
$$

The corresponding Lyapunov difference is given by

$$
\Delta V(k)=V(x(k+1), K(k+1))-V(x(k), K(k))
$$

During the manipulations, we let

$$
\begin{aligned}
& K(k+1)=K(k)-Q \hat{F}(x(k)) x^{T}(k) Y, \\
& \hat{F}(x(k))=B^{T} P\left(B K(k)+A_{c}\right) x(k),
\end{aligned}
$$

Next, add and subtract $\gamma^{2} w^{T}(k) w(k)$ and $x^{T}(k) K_{g}^{T} B^{T} P B K_{g} x(k)$ to and from (92), apply the conditions (87) to (89), and the fact $\operatorname{trx} y^{T}=y^{T} x, \forall x, y \in R^{n}$. In addition, assume that 


$$
\hat{R} \geq \hat{F}^{T}(x(k)) Q \hat{F}(x(k)) Y-2|\Delta A|_{2}^{2} P,
$$

where is a symmetric positive definite matrix. The resulting Lyapunov difference then becomes

$$
\Delta V(k) \leq-x^{T}(k) R x(k)+\gamma^{2} w^{T}(k) w(k) .
$$

Now, by summing (92) over $k \geq 0$ meets the nonexpansivity constraint given in Theorem 4.1. This completes the proof. Next, (93) could be rewritten as

$$
K(k+1)=K(k)-Q B^{T} P\left(B K(k)+A_{c}\right) x(k) x^{T}(k) Y,
$$

Furthermore, let $Q=q^{2}\left|B_{s}\right|^{-1}\left|B_{s}\right|^{-1}, K(k)=\left|B_{s}\right| K(k)$, and apply (15), (17). By similar procedure as in Corollary 3.2, (96) becomes (90). The closed-loop system, given by (86), (81), and (90), equivalent to

$$
x(k+1)=\left(A+B_{0} K(k)\right) x(k)+D w(k),
$$

\subsection{Adaptive Stabilization for Nonlinear Discrete-time Uncertain Systems}

The Lyapunov direct method gives sufficient conditions for Lyapunov stability of discrete-time dynamical systems. In this section, we begin by characterizing the problem of adaptive feedback control laws for nonlinear uncertain discrete time MIMO systems given by (Fu \& Cheng, 2004)

$$
x(k+1)=f(x(k))+G(x(k)) u(x(k)),
$$

where $w \in R^{d}, k \geq 1$, is the unknown exogenous disturbance, $x(k) \in R^{n}$ is the state vector, $u(k): R^{n} \rightarrow R^{m}$ is the control vector, $f: R^{n} \rightarrow R^{n}$ characterize system dynamics with uncertain entries, and $f(0)=0 . G: R^{n} \rightarrow R^{n \times m}$ is the input weighting matrix function. We assume that there exists a gain matrix $K_{g} \in R^{m \times s}, \hat{G}: R^{n} \rightarrow R^{m \times m}$, and vector $F: R^{n} \rightarrow R^{s}$, such that

$$
f_{c}(x(k))=f(x(k))+G(x(k)) \hat{G}(x(k)) K_{g} F(x(k)),
$$


is exponentially stable. We hereby state the main results of adaptive stabilization for nonlinear discrete-time uncertain systems.

\section{Theorem 5.1}

Consider the nonlinear discrete time system $G$ given by (98), where the system dynamics $f$ is uncertain, such that there exists a gain matrix $K_{g}$ and (99) is applied. Next, let $P_{1 u}: R^{n} \rightarrow R^{1 \times m}, P_{2 u}: R^{n} \rightarrow R^{m \times m}$, and the Lyapunov function $V_{s}$ is given by

$$
\begin{aligned}
& V_{s}(x(k+1))=V_{s}(f(x(k)))+P_{1 u}(x(k)) u(x(k)) \\
& +u^{T}(x(k)) P_{2 u}(x(k)) u(x(k)),
\end{aligned}
$$

In addition, let $\Gamma: R^{n} \rightarrow R$ is a positive scalar function, $\ell: R^{n} \rightarrow R^{p}$ is output vector, then

$$
0=V_{s}\left(f_{c}(x(k))\right)-V_{s}(x(k))+\ell^{T}(x(k)) \ell(x(k))+\Gamma(x(k)),
$$

The adaptive feedback control law

$$
u(x(k))=\hat{G}(x(k)) K(k) F(x(k))
$$

with the update law

$$
\begin{aligned}
& K(k+1)=K(k)-\frac{1}{2} Q \hat{G}^{T}(x(k)) P_{1 u}(x(k)) F^{T}(x(k)) Y \\
& -Q \hat{G}(x(k)) P_{2 u}^{T}(x(k)) \hat{G}(x(k)) K(k) F(x(k)) F^{T}(x(k)) Y,
\end{aligned}
$$

where $Q>0$ and $Y>0$, guarantees that the closed-loop system, given by (98), (102), and (103), is globally asymptotically stable.

\section{Proof}

We first consider the Lyapunov function candidate

$$
\begin{aligned}
& V(x(k), K(k))=V_{s}(x(k)) \\
& +\operatorname{tr} Q^{-1}\left(K(k)-K_{g}\right) Y^{-1}\left(K(k)-K_{g}\right)^{T},
\end{aligned}
$$


such that $V\left(0, K_{g}\right)=0$, and $V(x(k), K(k))>0$ for all $(x(k), K(k)) \neq\left(0, K_{g}\right)$. In addition, $V(x(k), K(k))$ is radially unbounded. Furthermore, assume that $V(\bullet, K(k))$ and $K(k)$ are continuous in $x(k)$ for $k \geq 1$. The corresponding Lyapunov difference is given by

$$
\Delta V(k)=V(x(k+1), K(k+1))-V(x(k), K(k)) .
$$

Next, consider the update law

$$
\begin{aligned}
& K(k+1)=K(k)-Q \hat{F}(x(k)) F^{T}(x(k)) Y, \\
& \hat{F}(x(k))=\frac{1}{2} \hat{G}^{T}(x(k)) P_{1 u}^{T}(x(k)) \\
& +\hat{G}^{T}(x(k)) P_{2 u}(x(k)) \hat{G}(x(k)) K(k) F(x(k)),
\end{aligned}
$$

and apply the fact $\operatorname{tr} x y^{T}=y^{T} x, \forall x, y \in R^{n}$, then the Lyapunov difference be comes

$$
\begin{aligned}
& \Delta V(k)=V_{s}(f(x))-V_{s}(x)-F^{T}(x) K_{g}^{T} \hat{G}(x) P_{2 u}(x) \hat{G}(x) K_{g} F(x) \\
& -F^{T}(x) K^{T}(x) \hat{G}(x) P_{2 u}(x) \hat{G}(x) K(x) F(x) \\
& +2 F^{T}(x) K_{g}^{T} \hat{G}(x) P_{2 u}(x) \hat{G}(x) K(x) F(x) \\
& +\left[F^{T}(x) Y F(x)\right] \hat{F}(x) Q \hat{F}(x) .
\end{aligned}
$$

Furthermore, we select

$$
\begin{aligned}
& \Gamma(x(k))=\left[F^{T}(x(k)) Y F(x(k))\right] \hat{F}^{T}(x(k)) Q \hat{F}(x(k)), \\
& P_{2 u}(x(k))=G^{T}(x(k)) P_{n}^{T} P_{n} G(x(k)), \quad P_{n} \in R^{n \times n} .
\end{aligned}
$$

After some manipulations, the resulting Lyapunov difference becomes

$$
\begin{aligned}
& \Delta V(k)=-\ell^{T}(x(k)) \ell(x(k))- \\
& \left|P_{n} G(x(k)) \hat{G}(x)\left(K(k)-K_{g}\right) F(x)\right|_{2}^{2} \\
& \quad \leq-\ell^{T}(x(k)) \ell(x(k)) .
\end{aligned}
$$


where $|\bullet|_{2}^{2}$ is Euclidean norm. This proves that the closed loop system is asymptotically stable, if $\ell(x(k)) \neq 0, k \geq 0$, then $x(k) \rightarrow 0$ as $k \rightarrow \infty, \forall x(0) \in R^{n}$. Finally, combining (106), (103) can therefore be obtained.

Specifically, if $P_{1 u}(x(k))=2 P_{2 u}(x(k)) \hat{G}(x(k)) K(k) F(x(k))$ then (103) can be otained

$$
\begin{aligned}
& K(k+1)=K(k)-2 Q \hat{G}^{T}(x(k)) P_{2 u}(x(k)) \\
& \hat{G}(x(k)) K(k) F(x(k)) F^{T}(x(k)) Y,
\end{aligned}
$$

Note that the adaptive control law (103) or (109) do not require explicit knowledge of the matrix $K_{g}$ and the system dynamics. Next, we extend the above result to the uncertain system given by

$$
x(k+1)=f(x(k))+B u(x(k))
$$

where $B$ satisfies (15) is the sign definite matrix with unknown entries. We state without proof the following results.

\section{Corollary 5.1}

Consider the nonlinear discrete-time uncertain system $G$ given by (110). Assume that there exists a gain matrix $K_{g}$, such that $f_{c}(x(k))=f(x(k))+B K_{g} F(x(k))$ is exponentially stable. Next, $P_{1 u}: R^{n} \rightarrow R^{1 \times m}$ and $P_{2 u}: R^{n} \rightarrow R^{m \times m}$, such that Lyapunov function $V_{s}$ is given by

$$
\begin{aligned}
& V_{s}(x(k+1))=V_{s}(x(k))+P_{1 u}(x(k)) u(x(k)) \\
& +u^{T}(x(k)) P_{2 u}(x(k)) u(x(k)),
\end{aligned}
$$

Furthermore, let $\Gamma: R^{n} \rightarrow R$ is a positive scalar function, $\ell: R^{n} \rightarrow R^{p}$ is output vector, and (101) is satisfied. The adaptive feedback control law

$$
u(x(k))=K(k) F(x(k)),
$$

with the normalized update law

$$
\begin{aligned}
& K(k+1)=K(k)-q^{2} B_{0}^{T} P[x(k) \\
& \left.+B_{0} K(k) F(x(k))\right] F^{T}(x(k)) Y,
\end{aligned}
$$


where $K(k)=\left|B_{s}\right| K(k), q>0$ and $Y>0$, guarantees that the closed-loop system, given by (110), (112), and (113), can be rewritten as

$$
x(k+1)=f(x(k))+B_{0} K(k) x(k),
$$

is Lyapunov stable.

Note that Corollary 5.1 implies we may have different update law by different choice of $P_{1 u}$. By the end of this section, we can further extend the results from above to linear uncertain systems given as following

$$
x(k+1)=A x(k)+B u(x(k)),
$$

where $(A, B)$ be controllable pair. Next, assume there exists a gain matrix $K_{g}: R^{m \times n}$, such that $A_{c}=A+B K_{g}$ is exponentially stable, and let $\Delta A=A_{c}-A$ is bounded, and the norm $|\Delta A|$ indicates the system dynamics $A$ deviates from the stable solution $A_{c}$.

\section{Corollary 5.2}

Consider the linear discrete-time uncertain system given by (115). Furthermore, let $R \in R^{n \times n}$ and $P \in R^{n \times n}$ are positive definite matrices, $\Gamma: R^{n} \rightarrow R$ is a positive scalar function, such that the Lyapunov function

$$
P=A_{c} P A_{c}+R-\Gamma(x(k)),
$$

with the assumption that $R \geq|\Delta A|^{2}+\Gamma(x(k))$, where $x(k)$ is the solution. Then the adaptive feedback control law $u(x(k))=K(k) x(k)$ with the normalized update law

$$
K(k+1)=K(k)-q^{2} B_{0}^{T} P\left[A_{c}+B_{0} K(k)\right] x(k) x^{T}(k) Y,
$$

where $K(k)=\left|B_{s}\right| K(k), Q>0$ and $Y>0$ guarantees that the closed-loop system, given by (115), (117), can be rewritten as

$$
x(k+1)=A x(k)+B_{0} K(k) x(k),
$$

is Lyapunov stable. 


\section{Proof}

The result is a direct extension of Theorem 5.1 and Cororllary 5.1. Specifically, we consider the Lyapunov candidate

$$
\begin{aligned}
& V(x(k), K(k))=x^{T}(k) P x(k) \\
& +\operatorname{tr} Q^{-1}\left(K(k)-K_{g}\right) Y^{-1}\left(K(k)-K_{g}\right)^{T},
\end{aligned}
$$

Next, let $R \geq|\Delta A|^{2}+\Gamma(x(k))$, normalized adaptive law $K(k)=\left|B_{s}\right| K(k)$, $Q=q^{2}\left|B_{s}\right|^{-1}\left|B_{s}\right|^{-1}, \Gamma(x(k))=\hat{F}^{T}(x(k)) Q \hat{F}(x(k))$, and

$$
\hat{F}(x(k))=B^{T} P A_{c} x(k)+B^{T} P B K(k) x(k) .
$$

Furthermore, we can substitute (117) into (115), the closed-loop form can be rewritten as (118).

\section{Numerical Examples}

In this section we illustrate the utility of the proposed direct adaptive control frameworks, both discrete-time and continuous-time, in the control problems of chaotic oscillator (Loria et al. 1998), one-link rigid robotic manipulator given by (Zhihong et al., 1998), and flexible joint robot manipulator (de LeònMorales et al., 2001), (Haddad \& Hayakawa, 2002).

\subsection{The van der Pol oscillator}

The first example is a well known perturbed van der Pol equation used to model electrical circuit with triode valve (Loria et al. 1998), and given as following

$$
\ddot{v}+\mu\left(1-v^{2}\right) \dot{v}+v=u+q \cos (\omega t),
$$

where the parameters specifically chosen as $\mu=5, q=5$, and $\omega=2.463$, which exhibits chaotic behaviour, and $u$ is control input. Next, let state space form with $x=[v, \dot{v}]^{T}=\left[x_{1}, x_{2}\right]^{T}$, (121) be rewritten as 
$f(x)=\left[\begin{array}{c}x_{2} \\ -\mu\left(1-x_{1}^{2}\right) x_{2}-x_{1}\end{array}\right], G(x)=\left[\begin{array}{l}0 \\ 1\end{array}\right], J(x)=1, \theta_{1}=\left[\begin{array}{l}0 \\ q\end{array}\right], \bar{w}(x, t)=\cos (\omega t)$.

Next, let

$$
F(x)=\left[\begin{array}{c}
x_{2} \\
x_{1}^{2} x_{2}
\end{array}\right], K_{g}=\left[\begin{array}{c}
\mu-\beta \\
-\mu
\end{array}\right], A_{c}=\left[\begin{array}{cc}
0 & 1 \\
-1 & -\beta
\end{array}\right]
$$

Specifically, we chose

$R=\left[\begin{array}{c}0.005 \\ 12.5\end{array}\right], Z=0.5, \beta=0.8, Y=1, P_{1}=\left[\begin{array}{cc}100 & 0 \\ 0 & 25\end{array}\right], x(0)=\left[\begin{array}{l}1 \\ 1\end{array}\right], K(0)=\left[\begin{array}{ll}0 & 0\end{array}\right]$, and $P$ is the solution of Lyapunov equation

$$
A_{c}^{T} P+P A_{c}+R=0,
$$

By Corollary 2.1, the closed-loop system guarantees $x \rightarrow 0$ as $t \rightarrow \infty$, if $\bar{w}(x, t)=0$. Figure 1 shows the phase portrait of the controlled system. The adaptive controller regulate the perturbed system to the origin under no knowledge of system dynamics, matrix $K_{g}$, and disturbance, while the disturbance exist. Figures 2 illustrates the time response of the feedback gain $K$ and the control inputs.

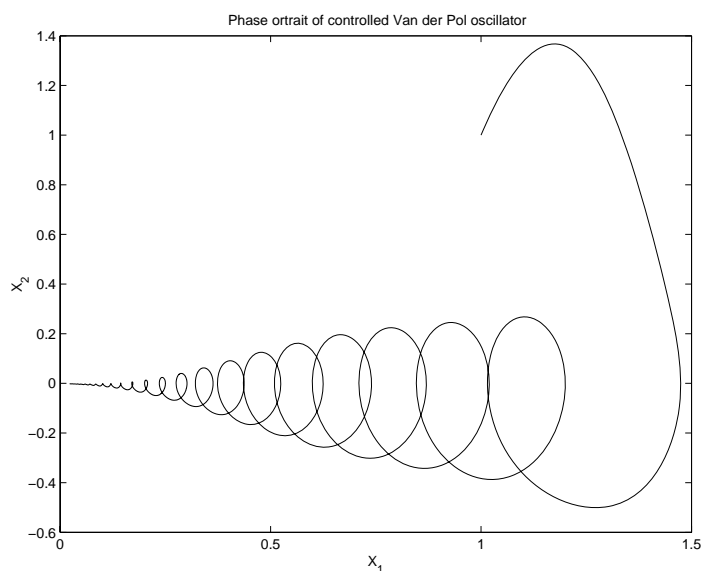

Figure 1 Phase Plot of perturbed van der pol equation
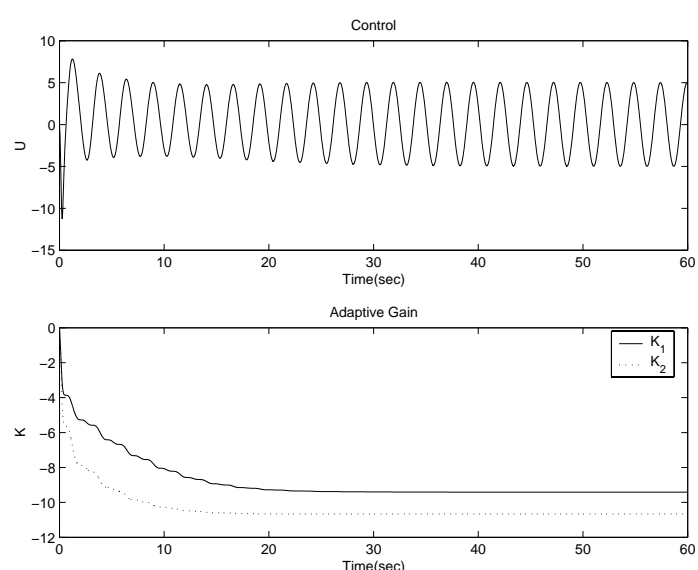

Figure 2 Control Signal and Adaptive gains

\subsection{One-Link Rigid Robot under Gravitation Field}

The dynamic equation of the one-link rigid robot placed on a tilted surface with an fixed angle $\theta$ is given by (Zhihong et al, 1998) 


$$
\left[\begin{array}{c}
\dot{q} \\
\ddot{q}
\end{array}\right]=\left[\begin{array}{cc}
0 & 1 \\
0 & -\frac{d}{m l^{2}}
\end{array}\right]\left[\begin{array}{l}
q \\
\dot{q}
\end{array}\right]+\left[\begin{array}{c}
0 \\
\frac{1}{m l^{2}}
\end{array}\right] u-\left[\begin{array}{c}
0 \\
\frac{g}{l}
\end{array}\right] \cos (q+\theta),
$$

and the reference model is defined as

$$
\left[\begin{array}{l}
\dot{q}_{r} \\
\ddot{q}_{r}
\end{array}\right]=\left[\begin{array}{cc}
0 & 1 \\
-16 & -8
\end{array}\right]\left[\begin{array}{l}
q_{r} \\
\dot{q}_{r}
\end{array}\right]+\left[\begin{array}{l}
0 \\
1
\end{array}\right] r
$$

Next, since the tracking error is defined as

$$
\left[\begin{array}{l}
e \\
\dot{e}
\end{array}\right]=\left[\begin{array}{l}
q \\
\dot{q}
\end{array}\right]-\left[\begin{array}{l}
q_{r} \\
\dot{q}_{r}
\end{array}\right]
$$

the tracking model can be formulated as

$$
\begin{aligned}
& {\left[\begin{array}{c}
\dot{e} \\
\ddot{e}
\end{array}\right]=\left[\begin{array}{cc}
0 & 1 \\
0 & \left.-\frac{d}{m l^{2}}\right]
\end{array}\right]\left[\begin{array}{l}
q \\
\dot{q}
\end{array}\right]+\left[\begin{array}{c}
0 \\
\frac{1}{m l^{2}}
\end{array}\right] u} \\
& +\left[\begin{array}{ccccc}
0 & 0 & 0 & 0 & 0 \\
16 & 1 & 1 & 1 & 1
\end{array}\right] \bar{w}\left[\begin{array}{c}
1 \\
8-\frac{d}{m l^{2}} \\
-\frac{g}{l} \cos (\phi) \\
\frac{g}{l} \sin (\phi) \\
1
\end{array}\right],
\end{aligned}
$$

where

$$
\bar{w}=\left[\begin{array}{ccccc}
q_{r} & 0 & 0 & 0 & 0 \\
0 & \dot{q}_{r} & 0 & 0 & 0 \\
0 & 0 & \cos (q) & 0 & 0 \\
0 & 0 & 0 & \sin (q) & 0 \\
0 & 0 & 0 & 0 & r
\end{array}\right] .
$$

Specifically, we chose 
$R=\left[\begin{array}{cc}100 & 0 \\ 0 & 500\end{array}\right], Z=8000, Y=6000, P_{1}=\left[\begin{array}{ccccc}10 & 0 & 0 & 0 & 0 \\ 0 & 0.1 & 0 & 0 & 0 \\ 0 & 0 & 0.02 & 0 & 0 \\ 0 & 0 & 0 & 0.05 & 0 \\ 0 & 0 & 0 & 0 & 0.02\end{array}\right]$,

$r=\sin (20 t)$

and $P$ is the solution of Lyapunov equation (121). In addition, let $m=l=d=1$ and $g=9.8$. Since (125) fits (13), and Corollary 2.1 can be directly applied. The initial conditions given $e(0)=\left[\begin{array}{ll}0 & 0\end{array}\right]^{T}$ and $K(0)=\left[\begin{array}{ll}0 & 0\end{array}\right]$. To demonstrate the robustness of the controller handle the uncertainty of the system dynamics, we introduce a changed to $m=0.8$ at time $t=0.5$ second. The simulation results, Figure 3 shows the states for each time step. The adaptive controller regulate the perturbed system to the origin under no knowledge of system dynamics, matrix $K_{g}$, and disturbance, while the disturbance exist. Figures 4 illustrates the time response of the control input, a constant force is applied to compensate the gravitation field. It shows that the controller can readapt the sudden change and stabilize the system.
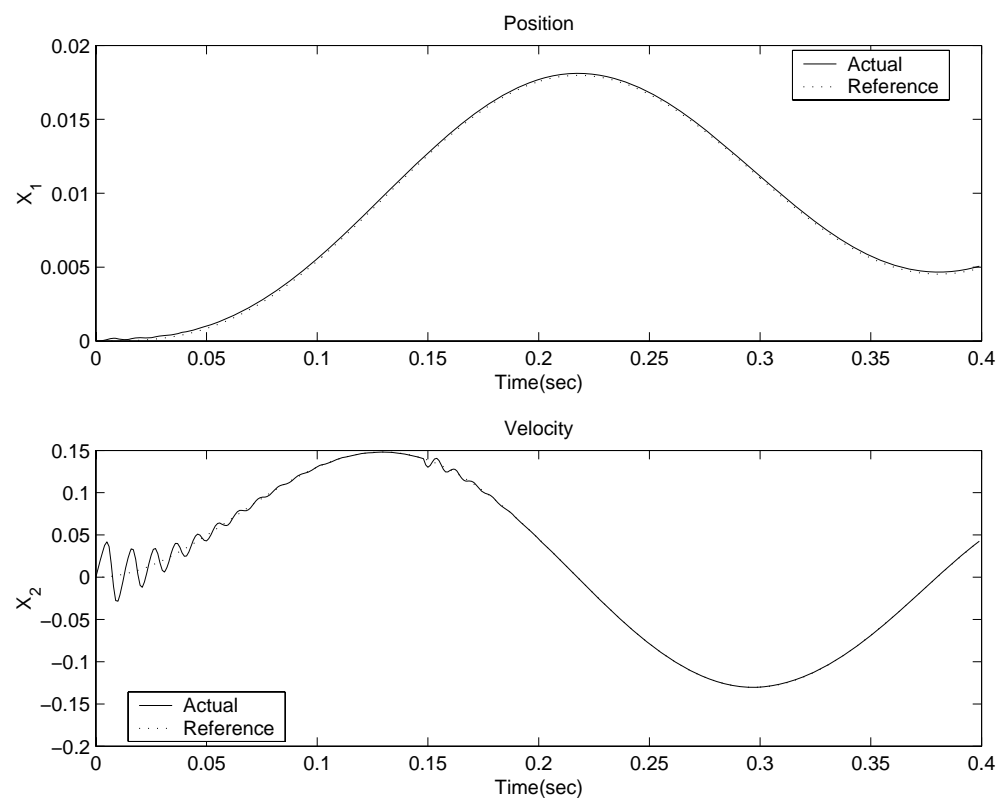

Figure 3. The states of one-link Rigid Robot 


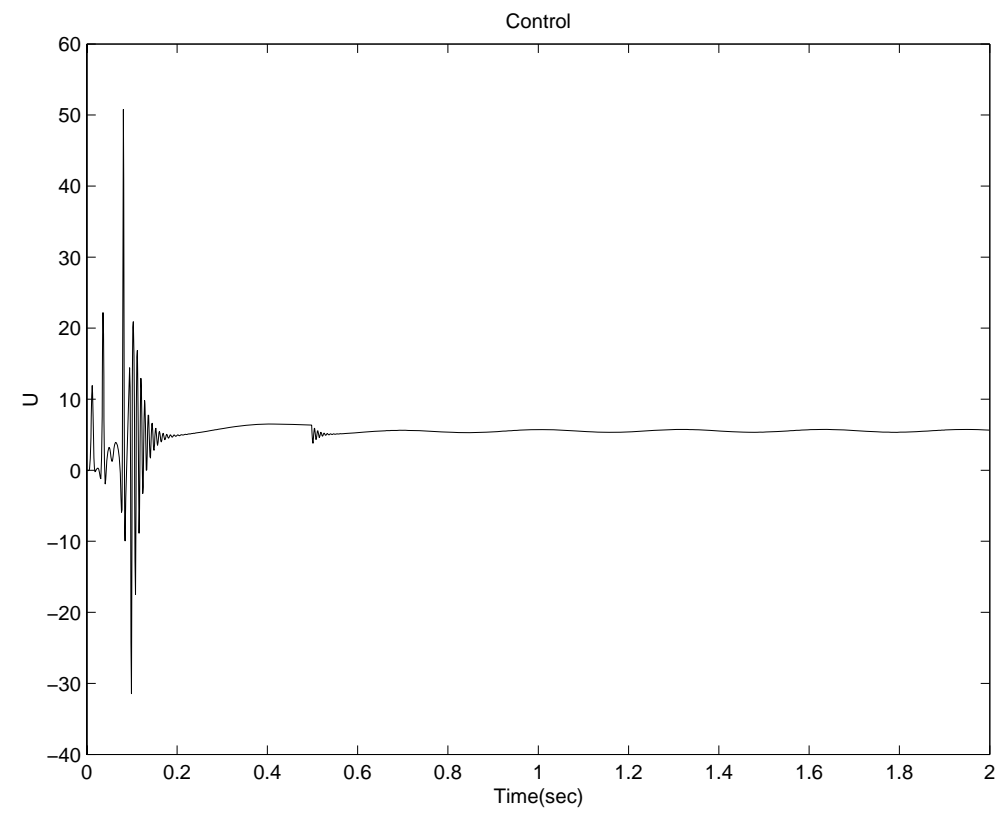

Figures 4. Control input

\subsection{Continuous-time Active Suspension System}

The dynamic equation for this quarter-car suspension is (Chantranuwathana \& Peng, 1999)

$$
\begin{aligned}
& \dot{x}=\left[\begin{array}{cccc}
1 & 0 & 0 & 1 \\
0 & 0 & -1 & 1 \\
0 & \frac{K_{s}}{m_{s}} & -\frac{C_{s}}{m_{s}} & \frac{C_{s}}{m_{s}} \\
-\frac{K_{u s}}{m_{u s}} & -\frac{K_{s}}{m_{u s}} & \frac{C_{s}}{m_{u s}} & -\frac{C_{u s}+C_{s}}{m_{u s}}
\end{array}\right] x \\
& -\left[\begin{array}{c}
0 \\
0 \\
\frac{1}{m_{s}} \\
-\frac{1}{m_{s}}
\end{array}\right] F_{a}+\left[\begin{array}{ll}
0 & 0 \\
0 & 0 \\
0 & 0 \\
1 & 1
\end{array}\right] \bar{w}\left[\begin{array}{c}
-\frac{K_{u s}}{m_{u s}} \\
\frac{C_{u s}}{m_{u s}}
\end{array}\right],
\end{aligned}
$$

where 
$x=\left[\begin{array}{c}x_{w} \\ x_{w}-x_{c} \\ \dot{x}_{c} \\ x_{w}\end{array}\right], \bar{w}=\left[\begin{array}{cc}x_{r} & 0 \\ 0 & \dot{x}_{r}\end{array}\right], x_{r}=\left\{\begin{array}{c}0.01 \sin (10 t), t \leq 0.8 \\ 0.07 \sin (5 t), 2<t \leq 2.2 \\ 0,0.8<t \leq 2 .\end{array}\right.$

$x_{w}, x_{c}$, and $x_{r}$ are displacements of wheel, vehicle, and road, $x_{w}-x_{c}$ is hydraulic piston displacement, $m_{s}=253 \mathrm{~kg}$ is sprung mass, $m_{u}=26 \mathrm{~kg}$ is unsprung mass, $C_{s}=348.5 \frac{\mathrm{N}}{\mathrm{m} \cdot \mathrm{sec}}$ is suspension damping, $C_{u s}=10 \frac{\mathrm{N}}{\mathrm{m} \cdot \mathrm{sec}}$ is tire damping, $K_{s}=12000 \frac{N}{m}$ is suspension stiffness, $K_{u s}=90000 \frac{N}{m}$ is tire stiffness, and $F_{a}$ is force of suspension actuator. Next, let $A_{c}$ is asymptotically stable.

$$
A_{c}=\left[\begin{array}{cccc}
1 & 0 & 0 & 1 \\
0 & 0 & -1 & 1 \\
0 & 0.05 & -1 & -0.05 \\
-0.1 & -5 & 1 & -5
\end{array}\right], \quad B_{0}=\left[\begin{array}{c}
0 \\
0 \\
1 \\
-1
\end{array}\right]
$$

First, we apply the framework of Corollary 2.1 and choosing the design matrices

$$
Y=\left[\begin{array}{cccc}
1 & 0 & 0 & 0 \\
0 & 0.5 & 0 & 0 \\
0 & 0 & 0.25 & 0 \\
0 & 0 & 0 & 0.5
\end{array}\right], \quad R=0.01 \cdot\left[\begin{array}{l}
1 \\
5 \\
7 \\
5
\end{array}\right], \quad Z=\left[\begin{array}{cc}
0.1 & 0 \\
0 & 0.001
\end{array}\right]
$$



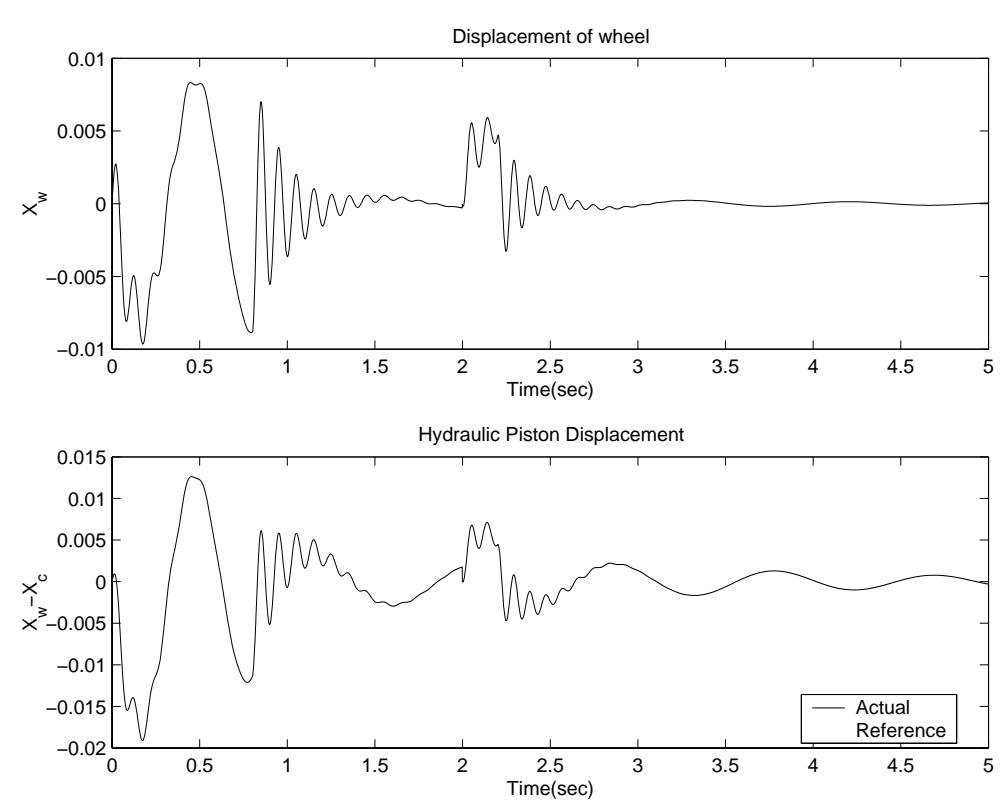

Figure 5 Displacement of wheel and Hydraulic pistion displacement
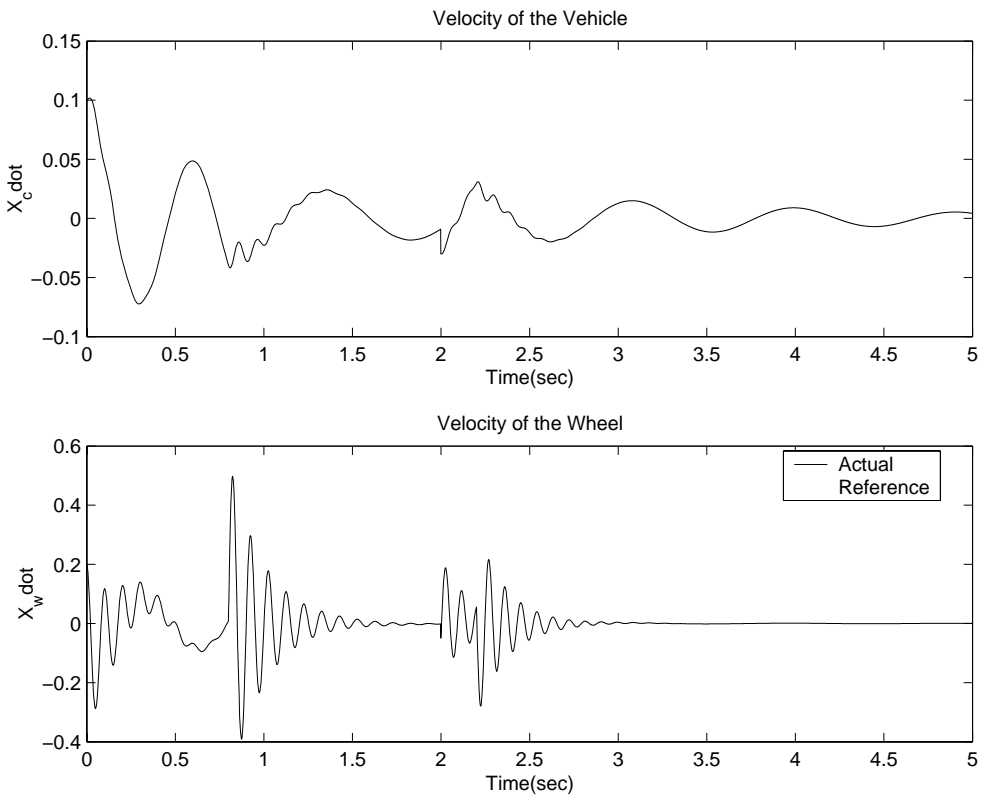

Figure 6 Velocity of vehicle and wheelhydraulic piston

where $P$ satisfies the lyapunov condition (121). The simulation start with $x(0)=\left[\begin{array}{llll}0 & 0 & 0.1 & 0.2\end{array}\right]^{T}$. At time $t=2 \mathrm{sec}$, the states are perturbed $x(2)=\left[\begin{array}{llll}0 & 0 & -0.03 & -0.05\end{array}\right]^{T}$, and the system parameters are changed to $m_{s}=213 \mathrm{~kg}, \quad m_{u}=20 \mathrm{~kg}, \quad C_{s}=320 \frac{\mathrm{N}}{\mathrm{m} \cdot \mathrm{sec}}, \quad C_{u s}=9 \frac{\mathrm{N}}{\mathrm{m} \cdot \mathrm{sec}}, \quad K_{s}=11500 \frac{\mathrm{N}}{\mathrm{m}}$, and 
$K_{u s}=85000 \frac{\mathrm{N}}{\mathrm{m}}$. The controller can re-adapt and stabilize the system in $5 \mathrm{sec}$ under no information of the system parameters, either the perturbation of the states. Figure 5 depicts displacement of wheel and hydraulic piston displacement versus the time, Figure 6 shows the velocity of vehicle and wheel versus time, Figure 7 and Figure 8 illustrate the control inputs and adaptive gains at each time step.

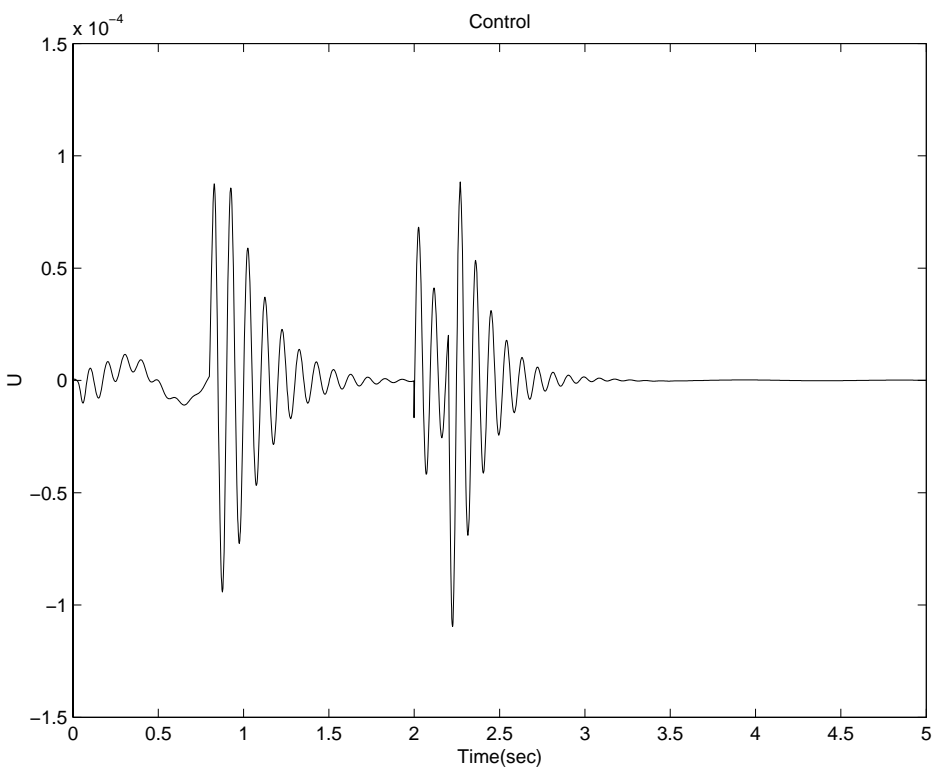

Figure 7 Control Input
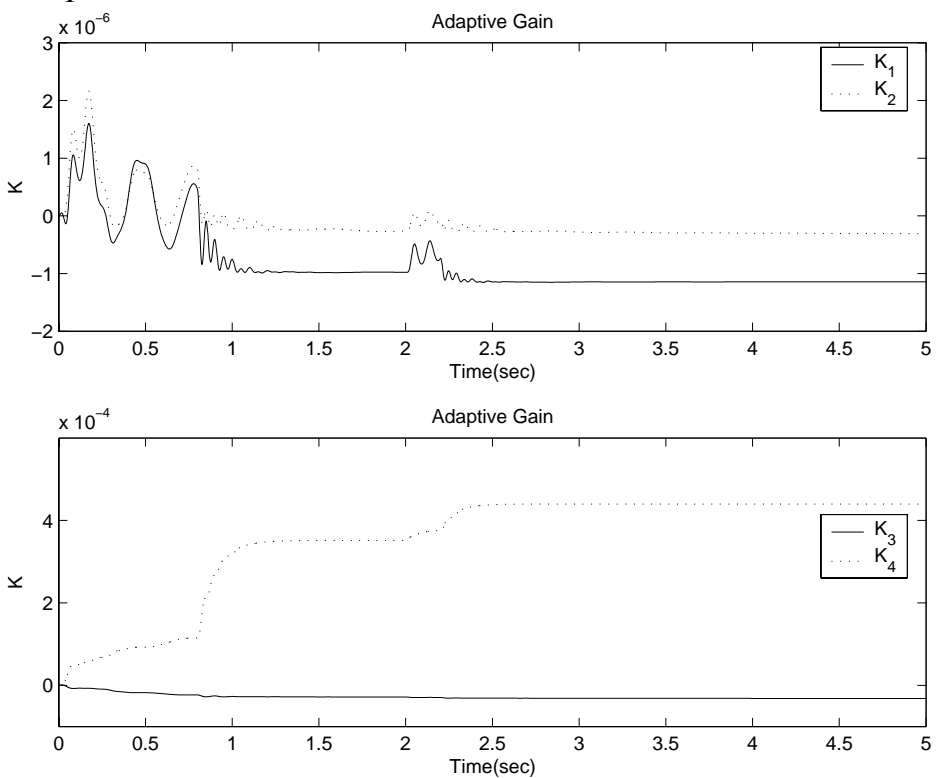

Figure 8 Adaptive Gains 


\subsection{Discrete-time Active Suspension System}

We use the quarter car model as the mathematical description of the suspension system, given by (Laila, 2003)

$$
\begin{aligned}
& x(k+1)=\left(\left[\begin{array}{cccc}
1 & T & 0 & 0 \\
-\frac{T \omega^{2}}{\rho+1} & 1 & \frac{T \rho \omega^{2}}{(\rho+1)^{2}} & 0 \\
0 & 0 & 1 & T \\
T \omega^{2} & 0 & -\frac{T \rho \omega^{2}}{\rho+1} & 1
\end{array}\right]\right. \\
& +\Delta(k)) x(k)-\left[\begin{array}{c}
T \\
0 \\
0 \\
0
\end{array}\right] d(k)-\left[\begin{array}{c}
0 \\
0 \\
0 \\
T(1+\rho)
\end{array}\right] u(x(k)),
\end{aligned}
$$

where

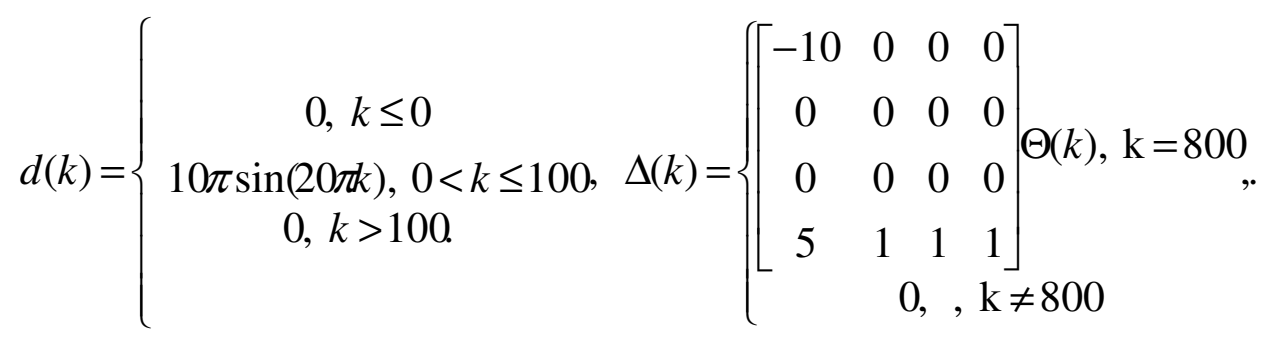

$\Theta(k)=|0.01 \sin (0.3 k)|$.

$x(k)=\left[\begin{array}{llll}x_{1}(k) & x_{2}(k) & x_{3}(k) & x_{4}(k)\end{array}\right]^{T}$, and $x_{1}$ is tire defection, $x_{2}$ is unsprung mass velocity, $x_{3}$ is suspension deflection, $x_{4}$ is sprung mass velocity, $\omega=20 \pi \frac{\mathrm{rad}}{\mathrm{sec}}$ and $\rho=10$ are unknown parameters, $T=0.001$ is sampling time, $d(k)$ is disturbance modeling the isolate bump with the bump height $A=0.01 \mathrm{~m}$, and $\Delta(k)$ is the perturbation on system dynamics. Next, let $A_{c}$ is asymptotically stable

$$
A_{c}=\left[\begin{array}{cccc}
1 & -1 & 0.75 & 1 \\
0.75 & 0.3 & -1 & -0.1 \\
0 & 0 & -0.1 & -0.5 \\
-0.1 & 0 & 0 & 0.1
\end{array}\right], \quad B_{0}=\left[\begin{array}{l}
0 \\
0 \\
0 \\
1
\end{array}\right],
$$

We apply the framework from Corollary 4.2 and choosing the design matrices 
$Y=0.03 \cdot\left[\begin{array}{cccc}2 & 0 & 0 & 0 \\ 0 & 1 & 0 & 0 \\ 0 & 0 & 4 & 0 \\ 0 & 0 & 0 & 1\end{array}\right], \quad R=\left[\begin{array}{cccc}1 & 0 & 0 & 0 \\ 0 & 4 & 0 & 0 \\ 0 & 0 & 9 & 0 \\ 0 & 0 & 0 & 0.82\end{array}\right], \quad q=0.05$
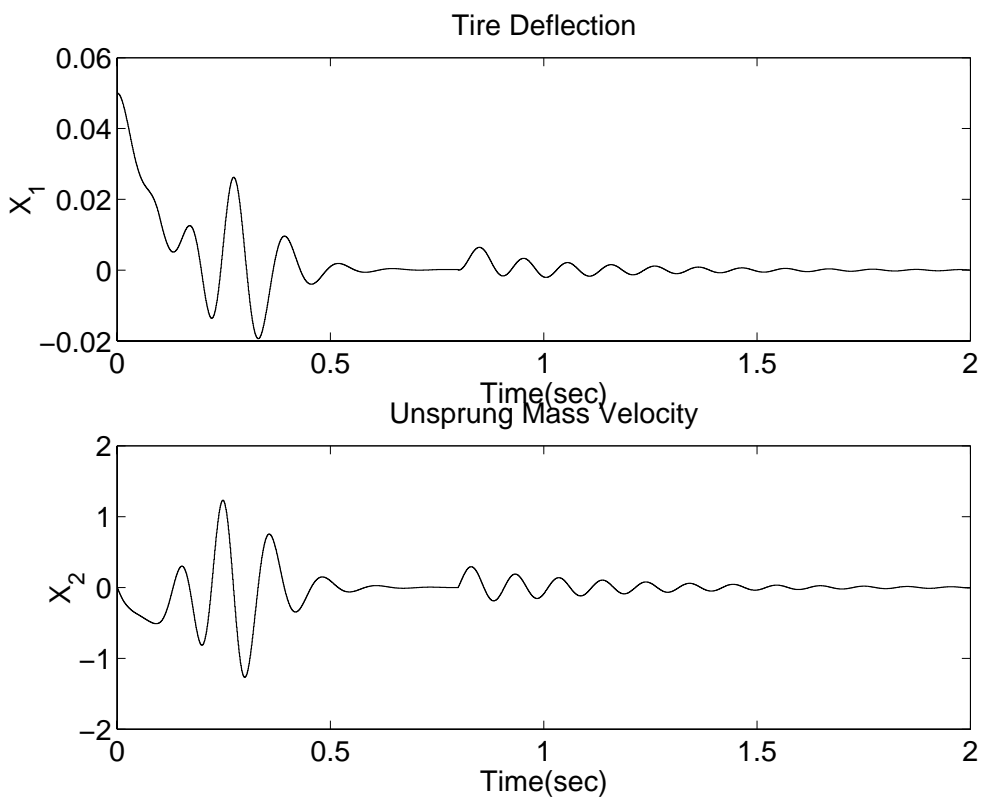

Figure 9 Tire defection and unsprung mass Velocity
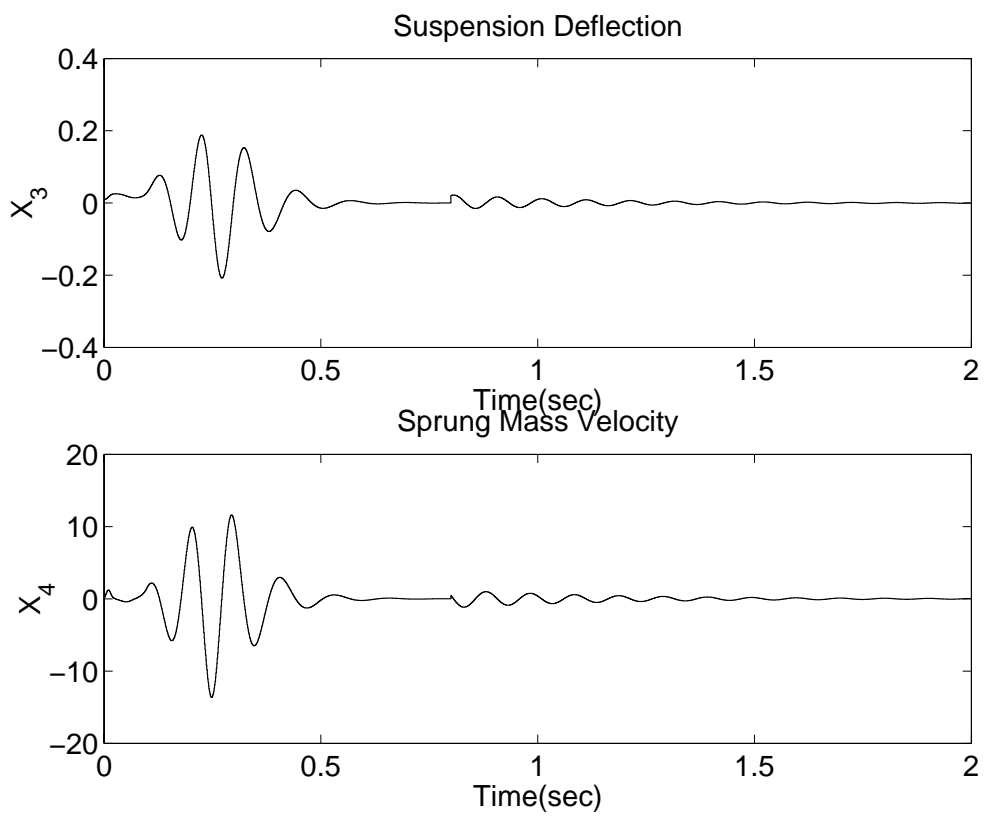

Figure 10 Suspension deflection and mass velocity 
$P$ satisfies the Lyapunov equation (121). The simulation start with $x(0)=\left[\begin{array}{llll}0.05 & 0 & 0.01 & 0\end{array}\right]^{T}$. To demonstrate the efficacy of the controller, the states are perturbed to $x(800)=\left[\begin{array}{llll}0 & 0 & 0.02 & 0.5\end{array}\right]^{T}$ at $k=800$, and the system parameters are changed to $\rho=4$. The controller stabilizes the system in $2 \mathrm{sec}$ under no information of the system changes, either the perturbation of the states. Figure 9 depicts tire defection and unsprung mass velocity versus the time steps, Figure 10 shows the suspension deflection and sprung mass velocity versus the time step, Figure 11 and Figure 12 illustrate the control inputs and adaptive gains at each time step.

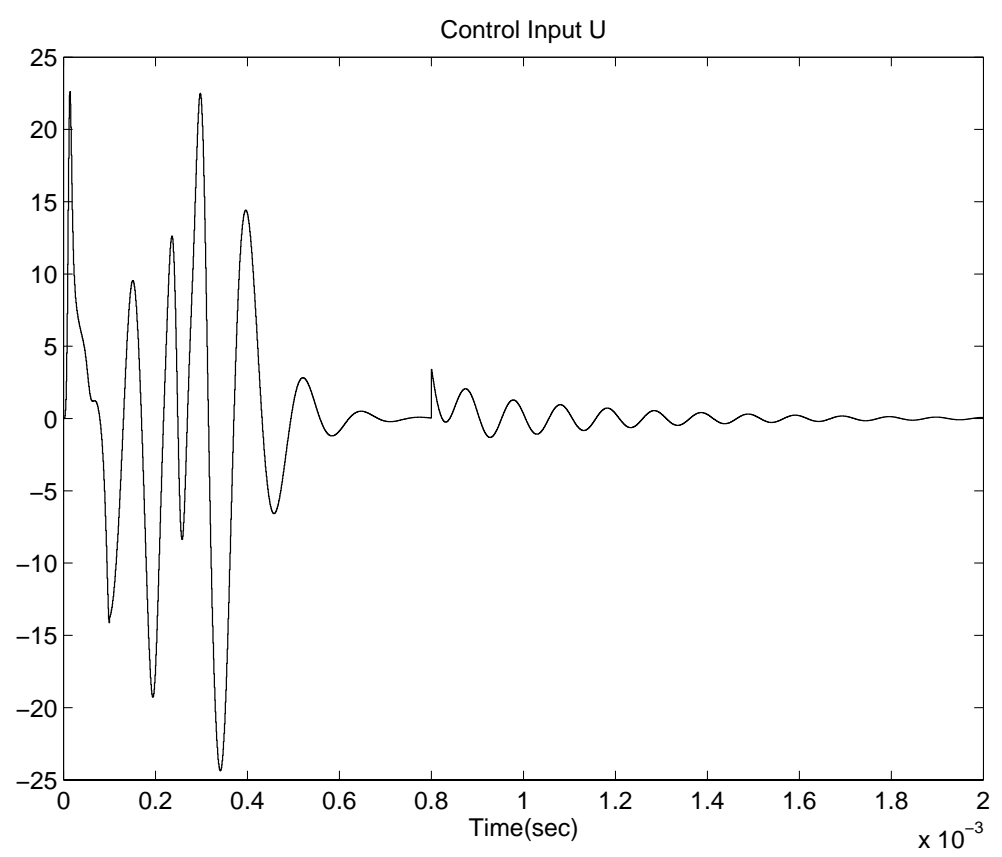

Figure 11 Control Input 


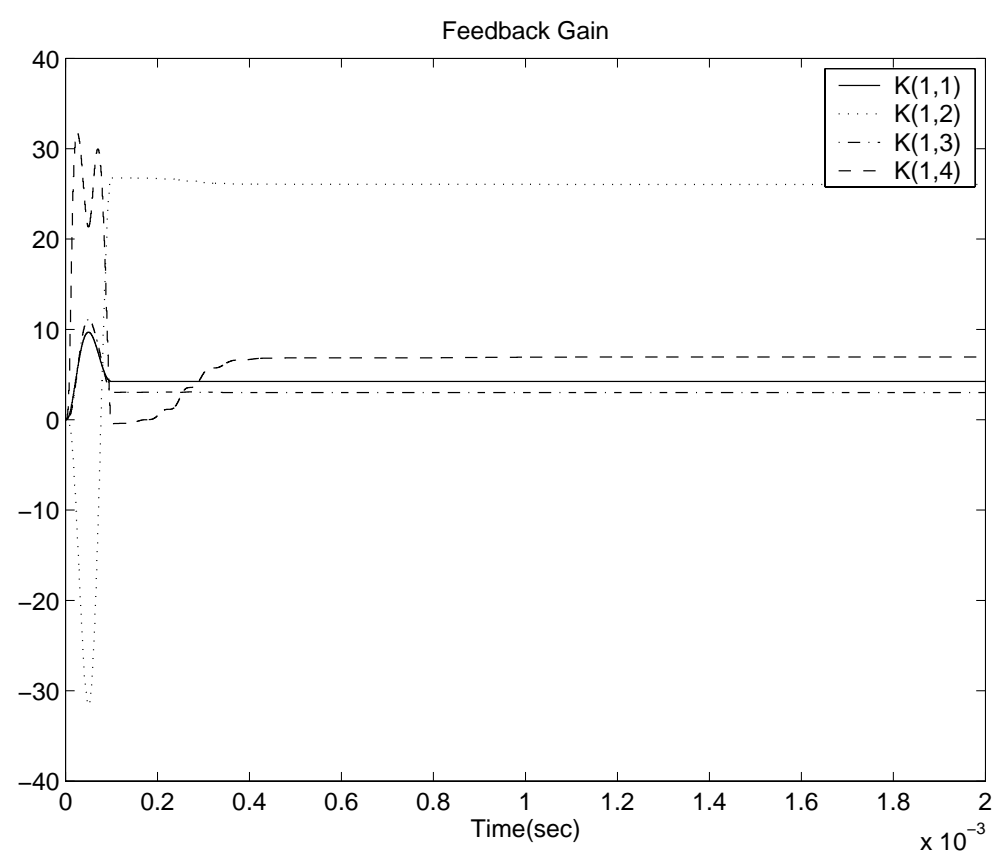

Figure 12 Adaptive Gains

\subsection{Nonlinear Discrete-time Uncertain System}

We consider the uncertain nonlinear discrete-time system in normal form given by (Fu \& Cheng, 2004); (Fu \& Cheng, 2005)

$$
x(k+1)=\left(\left[\begin{array}{c}
x_{2}(k) \\
a x_{1}^{2}(k)+b x_{2}(k) \cos \left(x_{2}(k)\right) \\
c x_{3}(k)+d x_{1}^{3}(k)
\end{array}\right]-\left[\begin{array}{ll}
0 & 0 \\
1 & 0 \\
0 & 1
\end{array}\right] u(x(k)),\right.
$$

where $a, b, c$, and $d$ are unknown parameters. Next, let $f_{c}(x(k))$ to be

$$
\begin{aligned}
f_{c}(x(k))= & A_{0} x(k)+\left[\begin{array}{c}
0 \\
a x_{1}^{2}(k)+b x_{2}(k) \cos \left(x_{2}(k)\right) \\
c x_{3}(k)+d x_{1}^{3}(k)
\end{array}\right] \\
& +\left[\begin{array}{c}
0 \\
B_{s}
\end{array}\right]\left|B_{s}\right|^{-1}\left(\Theta_{n} f_{u}(x(k))-\Theta f_{u}(x(k))+\Phi_{n} \hat{f}_{u}(x(k))\right),
\end{aligned}
$$




$$
\begin{aligned}
& f_{u}(x(k))=\left[\begin{array}{c}
x_{1}^{2} \\
x_{2}(k) \cos \left(x_{2}(k)\right) \\
x_{3}(k) \\
x_{1}^{3}(k)
\end{array}\right], \quad \hat{f}_{u}(x(K))=\left[\begin{array}{l}
x_{1}(k) \\
x_{2}(k)
\end{array}\right], \\
& F(x(k))=\left[\begin{array}{c}
x_{1}^{2}(k) \\
x_{2}(k) \cos \left(x_{2}(k)\right) \\
x_{3}(k) \\
x_{1}^{3}(k) \\
x_{1}(k) \\
x_{2}(k)
\end{array}\right]
\end{aligned}
$$

and $\Theta_{n}$ and $\Phi_{n}$ are chosen such that

$$
\Theta_{n} f_{u}(x(k))+\Phi_{n} \hat{f}_{u}(x(k))=\hat{A} x(k) .
$$

where $\hat{A} \in R^{2 \times 3}$ is arbitrary, such that

$$
f_{c}(x(k))=\left[\begin{array}{c}
\tilde{A}_{0} \\
\hat{A}
\end{array}\right] x(k)=A_{c} x(k),
$$

and $A_{c}$ is asymptotically stable, specifically, chose

$$
A_{c}=\left[\begin{array}{ccc}
0 & 1 & 0 \\
-0.5 & 0.4 & 0.1 \\
0.3 & -0.5 & 0.9
\end{array}\right], \quad B_{0}=\left[\begin{array}{ll}
0 & 0 \\
1 & 0 \\
0 & 1
\end{array}\right]
$$

First, we apply the update law (113) and choosing the design matrices $Y=0.1 I_{6}, R=0.2 I_{3}$, and $q=0.005$, where $P$ satisfies the Lyapunov condition $P=A_{c}^{T} P A_{c}+R$. The simulation start with $x(0)=\left[\begin{array}{lll}1 & 0.5 & -1\end{array}\right]^{T}$, and let $a=0.5$, $b=0.1, c=0.3$, and $d=0.5$. At time $k=19$, the states are perturbed $x(19)=\left[\begin{array}{lll}1 & -0.5 & 0.5\end{array}\right]^{T}$, and the system parameters are changed to $a=0.65$, $b=0.25, c=0.45$, and $d=0.55$. The controller does not have the information of the system parameters, either the perturbation of the states. Figure 13 - Figure 15 show the states versus the time step, Figures 16 shows the control inputs at each time step, and Figure 17 shows the update gains. The results indicate that the proposed controller can stabilize the system with uncertainty in 
the system parameters and input matrix. In addition, re-adapt system while perturbation occurs. The only assumption required is sign definiteness of the input matrix and disturbance weighting matrix.

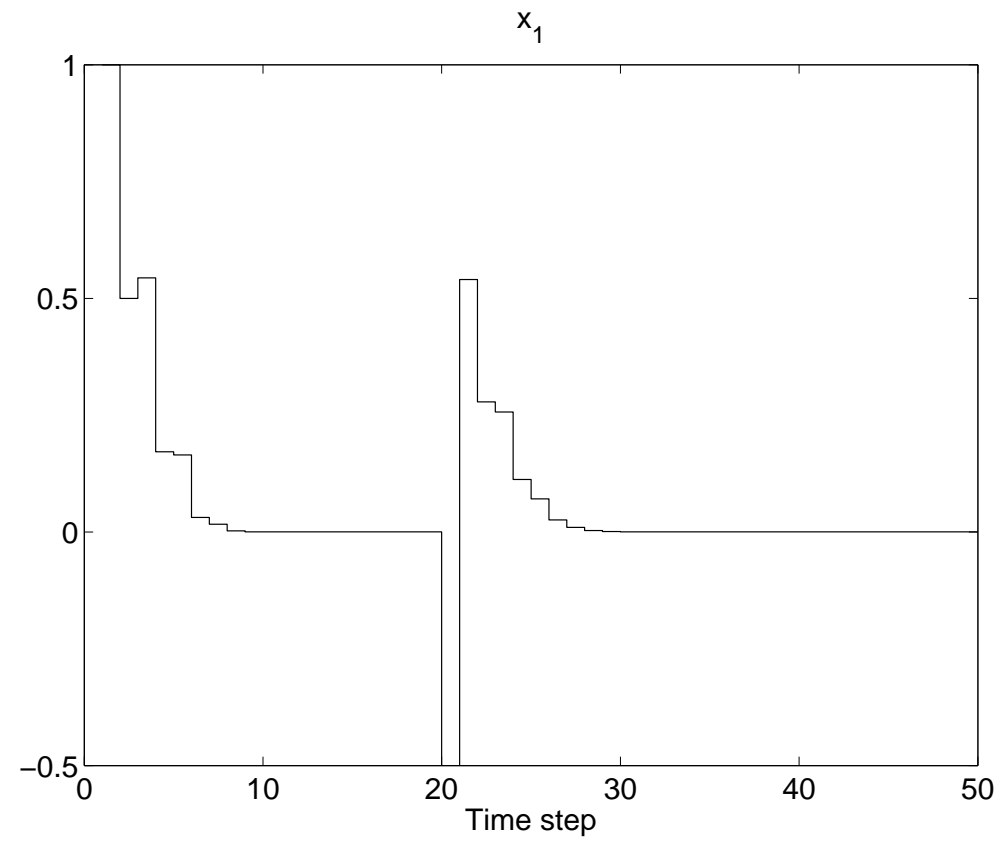

Figure $13 x_{1}$

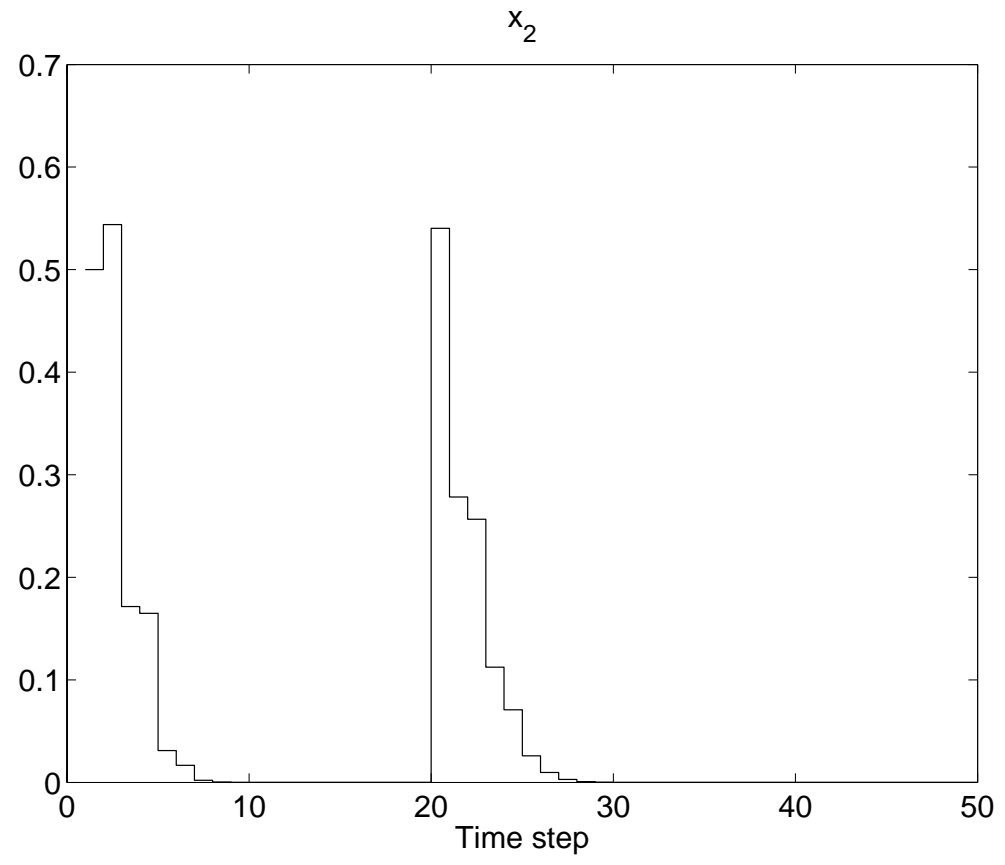

Figure 14. $x_{2}$ 


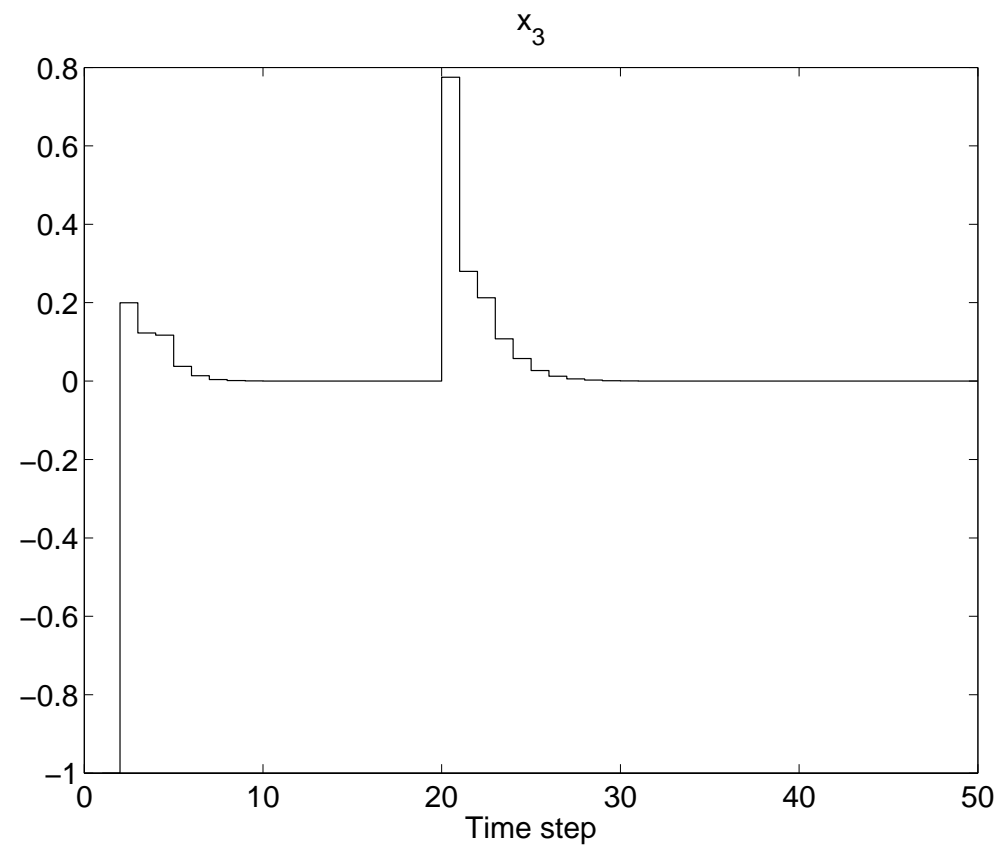

Figure 15. $x_{3}$
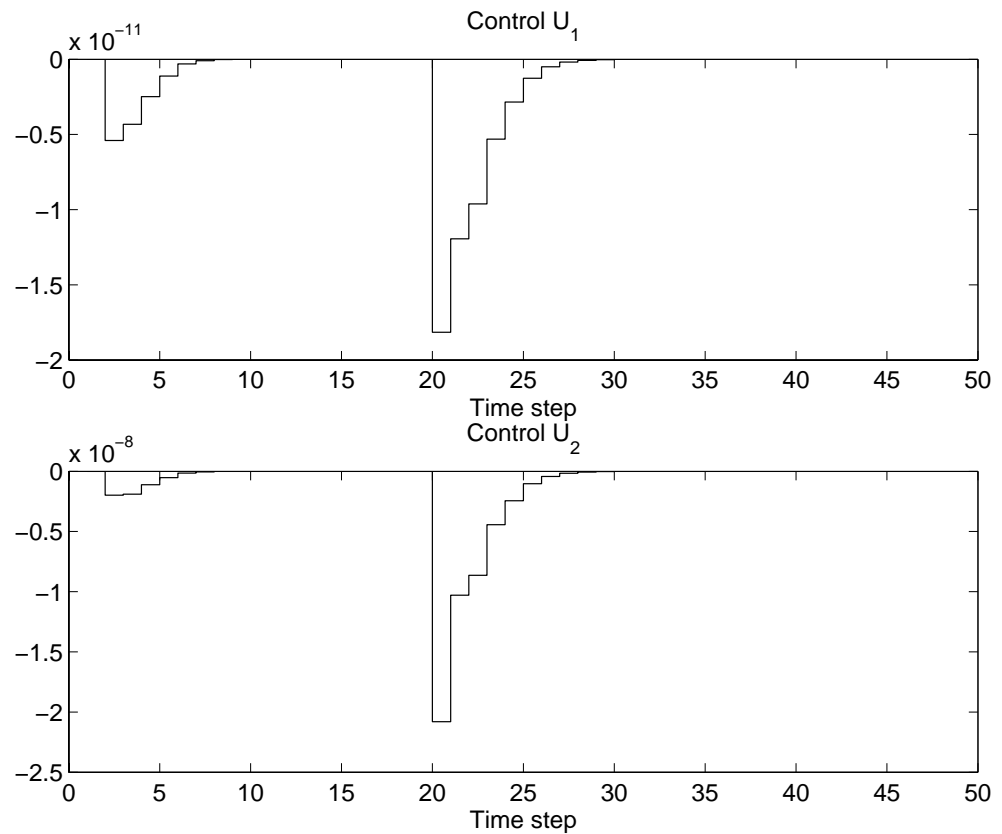

Figure 16. Control Signal 

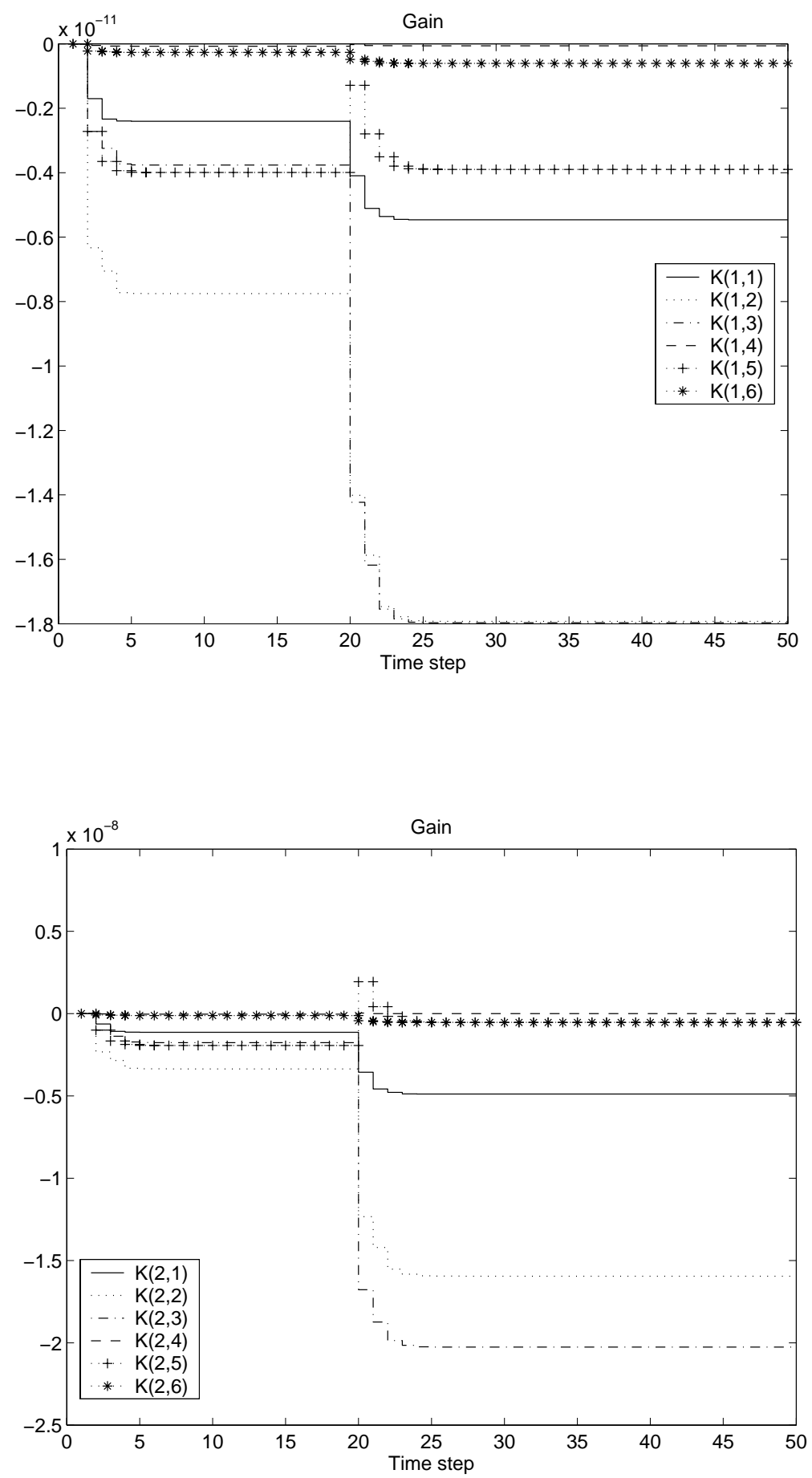

Figure 17. Update Gains 


\section{Conclusion}

In this Chapter, both discrete-time and continuous-time uncertain systems are investigated for the problem of direct adaptive control. Noted that our work were all Lyapunov-based schemes, which not only on-line adaptive the feedback gains without the knowledge of system dynamics, but also achieve stability of the closed-loop systems. We found that these approaches have following advantages and contributions:

1. We have successfully introduced proper Lyapunov candidates for both discrete-time and continuous-time systems, and to prove the stability of the resulting adaptive controllers.

2. A series of simple direct adaptive controllers were introduced to handle uncertain systems, and readapt to achieve stable when system states and parameters were perturbed.

3. Based on our research, we claim that a discrete-time counterpart of continuous-time direct adaptive control is made possible.

However, there are draw backs and require further investigation:

1. The nonlinear system is confined to normal form, which restrict the results of the proposed frameworks.

2. The assumptions of (63), (64), and (72) still limit our results.

Our future research directions along this field are as following:

1. Further investigate the optimal control application, i.e. to seek the adaptive control input $u \in L_{2}$ or $u \in l_{2}$, minimize certain cost function $f(u)$, such that not only a constraint is satisfied, but also satisfies Lyapunov hypothesis.

2. Stochastic control application, which require observer design under the extension of direct adaptive scheme.

3. Investigate alternative Lyapunov candidates such that the assumptions of (63), (64), and (72) could be released.

4. Application to ship dynamic control problems.

5. Direct adaptive control for output feedback problems, such as 


$$
\begin{aligned}
& x(k+1)=f(x(k))+G(x(k)) u(x(k))+J(x(k)) w(k), \\
& y(k)=H(x(k)) x(k)+I(x(k)) u(x(k)), \\
& u(k)=K(k) y(k)
\end{aligned}
$$

or

$\dot{x}=f(x(t))+G(x(t)) u(x(t))+J(x(t)) w(t)$,

$y=H(x(t)) x(t)+I(x(t)) u(x(t))$,

$u(t)=K(t) y(t)$

\section{References}

Bar-Kana, I. (1989), Absolute Stability and Robust Discrete Adaptive Control of Multivariable Systems, Control and Dynamic Systems, pp. 157-183, Vol. 31.

Chantranuwathana, S. \& Peng, H. (1999), Adaptive Robust Control for Active Suspension, Proceedings of the American Control Conference, pp. 1702-1706, San Diego, California, June, 1999.

de Leòn-Morales, J.; Alvarez-Leal, J. G.; Castro-Linares, R. \& Alvarez-Gallego, J. A. (2001), Control of a Flexible Joint Robot Manipulator via a Nonlinear Control-Observer Scheme, Int. J. Control, vol. 74, pp. 290-302 .

Fu, S. \& Cheng, C. (2003, a), Direct Adaptive Control Design for Reachable Linear Discrete-time Uncertain Systems, in Proceedings of IEEE International Symposium on Computational Intelligence in Robotics and Automation, pp. 1306-1310, Kobe, Japan, July, 2003.

Fu, S. \& Cheng, C. (2003, b), Direct Adaptive Control Design for Linear Discrete-time Uncertain Systems with Exogenous Disturbances and $\ell_{2}$ Disturbances, in Proceedings of IEEE International Symposium on Computational Intelligence in Robotics and Automation, pp. 1306-1310, Kobe, Japan, July, 2003.

Fu, S. \& Cheng, C. (2004, a), Adaptive Stabilization for Normal Nonlinear Discrete-time Uncertain Systems, in Proceedings of Fifth ASCC, pp. 20422048, Melbourne, Australia, July, 2004.

Fu, S. \& Cheng, C. (2004, b), Direct Adaptive Control for a Class of Linear Discrete-time Systems, in Proceedings of Fifth ASCC, pp. 172-176, Melbourne, Australia, July, 2004. 
Fu, S. \& Cheng, C. (2004, c), Direct Adaptive Feedback Design for Linear Discrete-time Uncertain Systems, Asia Journal of Control, Vol. 6, No. 3, pp. 421-427.

Fu, S. \& Cheng, C. (2005, a), Robust Direct Adaptive Control of Nonlinear Uncertain Systems with Unknown Disturbances, Proceedings of American Automatic Control Conference, pp. 3731-3736, Portland, Oregon, June , 2005.

Fu, S. \& Cheng, C. (2005, b), Direct Adaptive Control Designs for Nonlinear Discrete-time Systems with Matched Disturbances, Proceedings of IEEE International Conference on Mechatronics, pp. 881-886, Taipei, Taiwan, July 2005.

Fukaom, T.; Yamawaki, A. \& Adachi, N. (1999), Nonlinear and $H_{\infty}$ Control of Active Syspension Systems with Hydraulic Actuators, Proceedings of the 38th IEEE CDC, pp. 5125-5128, Phoenix, Arizona, December, 1999.

Guo, L. (1997), On Critical Stability of Discrete-time Adaptive Nonlinear Control, IEEE Transactions on Automatic Control, pp. 1488-1499, Vol. 42.

Haddad, W. \& Hayakawa, T. (2002). Direct Adaptive Control for Nonlinear Uncertain Systems with Exogenous Disturbances, Journal of Signal Processing and Adaptive Control, Vol. 16, pp 151-172.

Haddad, W.; Hayakawa, T. \& Leonessa, A. (2002), Direct Adaptive Control for Discrete-time Nonlinear Uncertain Dynamical Systems, in Proceedings of American Control Conference, pp. 1773-1778, Anchorage, May 2002.

Hitz, L. \& Anderson, B. D. O. (1969), Discrete Positive-real Functions and Their Application to System Stability, Proc. IEE, pp. 153-155, Vol. 116.

Johansson, R. (1989), Global Lyapunov Stability and Exponential Convergence of Direct Adaptive Control, Int. J. Control, pp. 859-869, Vol. 50.

Laila, D. S. (2003), Integrated Design of Discrete-time Controller for an Active Suspension System, Proceedings of the 42th IEEE CDC, pp. 6406-6411, Maui, Hawaii, December 2003.

Levin, A. \& Narendra, K. (1996), Control of Nonlinear Dynamical Systems using Neural Networks-Part II: Observability, Identification, and Control, IEEE Trans. Neural networks, Vol. 7, pp. 30-42.

Loria, A.; Panteley, E.; Nijmeijer, H. \& Fossen, T. (1998), Robust Adaptive Control of Passive Systems with Unknown Disturbances, IFAC NOLCOS, pp. 866-872, Enschede, The Netherlands, 1998.

Mareels, I. \& Polderman, J. (1996), Adaptive Systems An Introduction, Birkhauser. 
Venugopal, R. \& Bernstein, D. (1999), Adaptive Disturbance Rejection Using ARMARKOV System Representations, Proc. of the 36th IEEE CDC, pp. 1654-1658, San Diego, CA, December 1999.

Shibata, H.; Li, D.; Fujinaka, T. \& Maruoka, G. (1996), Discrete-time Simplified Adaptive Control Algorithm and its Application to a Motor Control, IEEE Industrial Electronics, pp. 248-253, Vol. 1.

Zhao, J. \& Kanellakopoulos I. (1997), Discrete-Time Adative Control of Output-Feedback Nonlinear Systems, in IEEE Conference on Decision and Control, pp. 4326-4331, San Diego, CA, December 1997.

Zhihong, M.; Wu, H. R. \& Palaniswami, M. (1998), An Adaptive Tracking Controller Using Neural Network for a Class of Nonlinear Systems, IEEE Transactions on Neural Network, Vol. 9, pp 947-954. 


\section{Manufacturing}

the Future

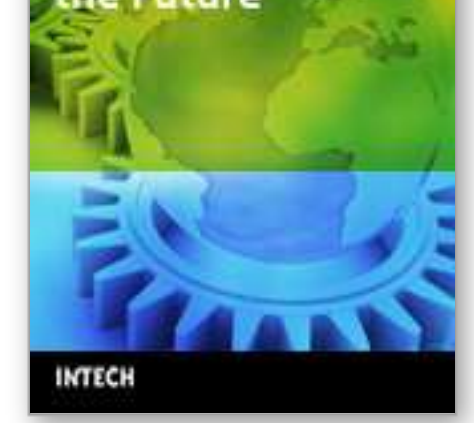

\section{Manufacturing the Future}

Edited by Vedran Kordic, Aleksandar Lazinica and Munir Merdan

ISBN 3-86611-198-3

Hard cover, 908 pages

Publisher Pro Literatur Verlag, Germany / ARS, Austria

Published online 01, July, 2006

Published in print edition July, 2006

The primary goal of this book is to cover the state-of-the-art development and future directions in modern manufacturing systems. This interdisciplinary and comprehensive volume, consisting of 30 chapters, covers a survey of trends in distributed manufacturing, modern manufacturing equipment, product design process, rapid prototyping, quality assurance, from technological and organisational point of view and aspects of supply chain management.

\section{How to reference}

In order to correctly reference this scholarly work, feel free to copy and paste the following:

Simon Hsu-Sheng Fu and Chi-Cheng Cheng (2006). On Direct Adaptive Control for Uncertain Dynamical Systems - Synthesis and Applications, Manufacturing the Future, Vedran Kordic, Aleksandar Lazinica and Munir Merdan (Ed.), ISBN: 3-86611-198-3, InTech, Available from:

http://www.intechopen.com/books/manufacturing_the_future/on_direct_adaptive_control_for_uncertain_dynam ical_systems_-_synthesis_and_applications

\section{INTECH}

open science | open minds

\section{InTech Europe}

University Campus STeP Ri

Slavka Krautzeka 83/A

51000 Rijeka, Croatia

Phone: +385 (51) 770447

Fax: +385 (51) 686166

www.intechopen.com

\section{InTech China}

Unit 405, Office Block, Hotel Equatorial Shanghai No.65, Yan An Road (West), Shanghai, 200040, China 中国上海市延安西路65号上海国际贵都大饭店办公楼 405 单元 Phone: +86-21-62489820

Fax: $+86-21-62489821$ 
(C) 2006 The Author(s). Licensee IntechOpen. This chapter is distributed under the terms of the Creative Commons Attribution-NonCommercial-ShareAlike-3.0 License, which permits use, distribution and reproduction for non-commercial purposes, provided the original is properly cited and derivative works building on this content are distributed under the same license. 